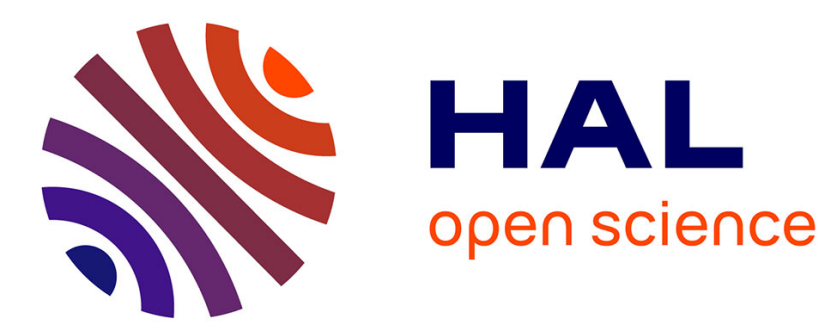

\title{
Fast Gossiping by Short Messages
}

Jean-Claude Bermond, Luisa Gargano, Adele Rescigno, Ugo Vaccaro

\section{To cite this version:}

Jean-Claude Bermond, Luisa Gargano, Adele Rescigno, Ugo Vaccaro. Fast Gossiping by Short Messages. SIAM Journal on Computing, 1998, 27 (4), pp.917-941. 10.1137/S0097539795283619 . hal03224084

\section{HAL Id: hal-03224084 \\ https://hal.science/hal-03224084}

Submitted on 11 May 2021

HAL is a multi-disciplinary open access archive for the deposit and dissemination of scientific research documents, whether they are published or not. The documents may come from teaching and research institutions in France or abroad, or from public or private research centers.
L'archive ouverte pluridisciplinaire HAL, est destinée au dépôt et à la diffusion de documents scientifiques de niveau recherche, publiés ou non, émanant des établissements d'enseignement et de recherche français ou étrangers, des laboratoires publics ou privés. 


\title{
FAST GOSSIPING BY SHORT MESSAGES*
}

\author{
JEAN-CLAUDE BERMOND ${ }^{\dagger}$, LUISA GARGANO $\ddagger$, ADELE A. RESCIGNO ${ }^{\ddagger}$, AND \\ UGO VACCARO $\ddagger$
}

\begin{abstract}
Gossiping is the process of information diffusion in which each node of a network holds a packet that must be communicated to all other nodes in the network. We consider the problem of gossiping in communication networks under the restriction that communicating nodes can exchange up to a fixed number $p$ of packets at each round. In the first part of the paper we study the extremal case $p=1$ and we exactly determine the optimal number of communication rounds to perform gossiping for several classes of graphs, including Hamiltonian graphs and complete $k$-ary trees. For arbitrary graphs we give asymptotically matching upper and lower bounds. We also study the case of arbitrary $p$ and we exactly determine the optimal number of communication rounds to perform gossiping under this hypothesis for complete graphs, hypercubes, rings, and paths. Finally, we investigate the problem of determining sparse networks in which gossiping can be performed in the minimum possible number of rounds.
\end{abstract}

1. Introduction. Gossiping (also called total exchange or all-to-all communication) in distribution systems is the process of distributing information known to each processor to every other processor of the system. This process of information dissemination is carried out by means of a sequence of message transmissions between adjacent nodes in the network.

The gossiping problem was originally introduced by the community of discrete mathematicians, to which it owes most of its terminology, as a combinatorial problem in graphs. Nonetheless, it was soon realized that, once cast in more realistic models of communication, gossiping is a fundamental primitive in distributed memory multiprocessor systems. There are a number of situations in multiprocessor computation, such as global processor synchronization, where gossiping occurs. Moreover, the gossiping problem is implicit in a large class of parallel computation problems, such as linear system solving, the discrete Fourier transform, and sorting, where both input and output data are required to be distributed across the network $[6,9,18]$. Due to the interesting theoretical questions it poses and its numerous practical applications, gossiping has been widely studied under various communication models. Hedetniemi, Hedetniemi, and Liestman [15] provide a survey of the area. Two more recent survey papers collecting the latest results are $[10,17]$. Readers could also profit from seeing the book [24].

† I3S, 'ĆNRS, Université de Nice, Sophia Antipolis, Bat ESSI, 650 Route de Colles, BP 145, 06903 Sophia Antipolis Cedex, France (bermond@alto.unice.fr). This research was partially supported by the French GDR/PRC Project PRS and by the French-Italian Bilateral Project Galileo. This research was partially performed while the author was visiting the Dipartimento di Informatica ed Applicazioni of the Università di Salerno.

¥ Dipartimento di Informatica ed Applicazioni, Università di Salerno, 84081 Baronissi (SA), Italy (1g@dia.unisa.it, rescigno@udsab.dia.unisa.it, uv@dia.unisa.it). This research was partially supported by the Italian Ministry of University and Scientific Research, Project: Algoritmi, Modelli di Calcolo e Strutture Informative and by the French-Italian Bilateral Project Galileo. 
The great majority of the previous work on gossiping has considered the case in which the packets known to a processor at any given time during the execution of the gossiping protocol can be freely concatenated and the resulting (longer) message can be transmitted in a constant amount of time; that is, it has been assumed that the time required to transmit a message is independent of its length. While this assumption is reasonable for short messages, it is clearly unrealistic when the size of the messages becomes large. Notice that most of the gossiping protocols proposed in the literature require the transmission, in the last rounds of the execution of the protocol, of messages of size $\Theta(n)$, where $n$ is the number of nodes in the network. Therefore, it would be interesting to have gossiping protocols that require only the transmission of bounded length messages between processors. In this paper we consider the problem of gossiping in communication networks under the restriction that communicating nodes can exchange up to a fixed number $p$ of packets in each round.

1.1. The model. Consider a communication network modeled by a graph $G=$ $(V, E)$ where the node set $V$ represents the set of processors of the network and $E$ represents the set of communication lines between processors.

Initially each node holds a packet that must be transmitted to any other node in the network by a sequence of calls between adjacent processors. During each call, communicating nodes can exchange up to $p$ packets, where $p$ is an a priori fixed integer. We assume that each processor can participate in at most one call at a time. Therefore, we can see the gossiping process as a sequence of rounds: during each round a disjoint set of edges (matching) is selected and the nodes that are end vertices of these edges make a call. This communication model is usually referred to as telephone model [15] or full-duplex 1-port $\left(F_{1}\right)[20]$. We denote by $g_{F_{1}}(p, G)$ the minimum possible number of rounds to complete the gossiping process in the network $G$ subject to the above conditions. Another popular communication model is the mail model [15] or half-duplex 1-port $\left(H_{1}\right)$ [20], in which in each round any node can either send a message to one of its neighbors or receive a message from it, but not simultaneously. The problem of estimating $g_{H_{1}}(p, G)$ has been considered in [4]. Analogous problems in bus networks have been considered in [11, 16]. Optimal bounds on $g_{H_{1}}(1, G)$ when the edges of $G$ are subject to random failures are given in [7]. Packet routing in interconnection networks in the $F_{1}$ model has been considered in [1].

1.2. Results. We first study the extremal case in which gossiping is to be performed under the restriction that communicating nodes can exchange exactly one packet in each round. We provide several lower bounds on the gossiping time $g_{F_{1}}(1, G)$ and we provide matching upper bounds for Hamiltonian graphs, complete trees, and complete bipartite graphs. For general graphs we provide asymptotically tight upper and lower bounds.

Subsequently, we study the case of arbitrary $p$ and we compute $g_{F_{1}}(p, G)$ exactly for complete graphs, hypercubes, rings, and paths. Our result for hypercubes allows us to improve the corresponding result in the $H_{1}$ model given in [4].

Finally, we investigate the problem of finding the sparsest networks in which gossiping can be performed in the minimum possible number of rounds.

All logarithms in this paper are to the base 2.

2. Gossiping by exchanging one packet at a time. In this section we study $g_{F_{1}}(1, G)$, that is, the minimum possible number of rounds to complete gossip in a graph $G$ under the condition that at each round communicating nodes can exchange 
exactly one packet.

In order to avoid overburdening the notation, we will simply write $g(G)$ to denote $g_{F_{1}}(1, G)$.

2.1. Lower bounds on $g(G)$. In this section we give some lower bounds on the time needed to complete the gossiping process.

LemmA 2.1. For any graph $G=(V, E)$, with $|V|=n$, let $\mu(G)$ be the size of a maximum matching in $G$; then

$$
g(G) \geq\left\lceil\frac{n(n-1)}{2 \mu(G)}\right\rceil .
$$

Proof. For any node $v \in V$ the packet initially resident in $v$ must reach each of the remaining $n-1$ nodes. Therefore, during the gossiping process, at least $n(n-1)$ packet transmissions must be executed over the edges of $G$. Since in each communication round at most $\mu(G)$ calls are performed and each call allows the transmission of two packets (one in each direction), the bound follows.

Lemma 2.2. Let $X \subset V$ be a vertex cutset of the graph $G=(V, E)$ whose removal disconnects $G$ into the connected components $V_{1}, \ldots, V_{d}$; then

$$
g(G) \geq\left\lceil\sum_{i=1}^{d} \frac{\max \left\{\left|V_{i}\right|, n-\left|V_{i}\right|\right\}}{\left|M_{X}\right|}\right\rceil,
$$

where $\left|M_{X}\right|$ is the size of a maximum matching $M_{X}$ in $G$ such that any edge in it has one endpoint in $X$ and the other in $V-X$.

Proof. Consider a component $V_{i}$, for some $1 \leq i \leq d$. Nodes in $V_{i}$ can receive the packets of nodes in $V-V_{i}$ only by means of calls between a node in $X$ and one in $V_{i}$; moreover, at least $n-\left|V_{i}\right|$ calls are needed between nodes in $X$ and nodes in $V_{i}$ to bring all packets in $V-V_{i}$ to nodes in $V_{i}$. Analogously, packets of nodes in $V_{i}$ can reach nodes in $V-V_{i}$ only by means of calls between a node in $X$ and one in $V_{i}$, and at least $\left|V_{i}\right|$ such calls are needed. Therefore, for each $i=1, \ldots, d$, at least $\max \left\{\left|V_{i}\right|, n-\left|V_{i}\right|\right\}$ calls must take place between nodes in $X$ and nodes in $V_{i}$. We then get that at least $\sum_{i=1}^{d} \max \left\{\left|V_{i}\right|, n-\left|V_{i}\right|\right\}$ calls are needed between nodes in $X$ and nodes in $V-X=\cup_{i=1}^{d} V_{i}$. Since at most $\left|M_{X}\right|$ such calls can take place during each round, we get the desired lower bound of

$$
\left\lceil\frac{\sum_{i=1}^{d} \max \left\{\left|V_{i}\right|, n-\left|V_{i}\right|\right\}}{\left|M_{X}\right|}\right\rceil
$$

on the time necessary to gossip in $G$.

Remark 2.1. The bound in Lemma 2.2 can sometimes be improved by observing that after the last call has been done between a node in some $V_{i}$ and a node in $X$, the last exchanged message has still to reach all the other nodes of $V_{i}$ (or of $V-V_{i}$ ). Therefore, we can add to the lower bound (2) the minimum of the eccentricities of the subgraphs induced by the $V_{i}$ 's and the $V-V_{i}$ 's.

Corollary 2.1. Let $\alpha(G)$ be the independence number of $G$; then

$$
g(G) \geq\left\lceil\frac{\alpha(G)(n-1)}{n-\alpha(G)}\right\rceil .
$$


Proof. Let $Y$ denote an independent set of $G$. Applying Lemma 2.2 with cutset $X=V-Y$ and connected components $V_{1}, \ldots, V_{|Y|}$, each consisting of just one element of $Y$, we get

$$
g(G) \geq\left\lceil\sum_{i=1}^{|Y|} \frac{n-\left|V_{i}\right|}{\left|M_{X}\right|}\right\rceil \geq\left\lceil\sum_{i=1}^{|Y|} \frac{n-\left|V_{i}\right|}{|X|}\right\rceil=\left\lceil\mid \sum_{i=1}^{|Y|} \frac{n-1}{n-|Y|}\right\rceil=\left\lceil\frac{|Y|(n-1)}{n-|Y|}\right\rceil .
$$

Choosing an independent set of maximum size $|Y|=\alpha(G)$ we get (3).

Let $T$ be a tree and $v$ one of its nodes; we indicate the connected components into which the node set of $T$ is split by the removal of $v$ by $V_{1}(v), \ldots, V_{\operatorname{deg}(v)}(v)$, ordered so that $\left|V_{1}(v)\right| \geq \cdots \geq\left|V_{\operatorname{deg}(v)}(v)\right|$.

COROLLARY 2.2. Let $T$ be a tree on $n$ nodes of maximum degree $\Delta=\max _{v \in V} \operatorname{deg}(v)$; then

$$
g(T) \geq \max _{v: \operatorname{deg}(v)=\Delta} L(v),
$$

where

$$
L(v)= \begin{cases}(\operatorname{deg}(v)-1) n+1 & \text { if }\left|V_{1}(v)\right| \leq n / 2, \\ (\operatorname{deg}(v)-2) n+1+2\left|V_{1}(v)\right| & \text { if }\left|V_{1}(v)\right|>n / 2 .\end{cases}
$$

Proof. Given a node $v$, Lemma 2.2 with $X=\{v\}$ gives

$$
\begin{aligned}
g(T) & \geq \sum_{i=1}^{\operatorname{deg}(v)} \max \left\{\left|V_{i}(v)\right|, n-\left|V_{i}(v)\right|\right\} \\
& =\sum_{i=2}^{\operatorname{deg}(v)} n-\left|V_{i}(v)\right|+ \begin{cases}\left|V_{1}(v)\right| & \text { if }\left|V_{1}(v)\right|>n / 2, \\
n-\left|V_{1}(v)\right| & \text { if }\left|V_{1}(v)\right| \leq n / 2,\end{cases} \\
& =L(v) .
\end{aligned}
$$

Direct computation shows that if $\operatorname{deg}(v)>\operatorname{deg}(w)$ then $L(v)>L(w)$, thus proving that the maximum is always attained at a node of maximum degree.

2.2. Upper bounds. In this section we will determine $g(G)$ exactly for several classes of graphs, including Hamiltonian graphs and complete $k$-ary trees. We will also provide good upper bounds for general graphs.

2.2.1. Hamiltonian graphs. We first note that in any graph $G=(V, E)$ the size of a maximum matching $\mu(G)$ is at most $\lfloor|V| / 2\rfloor$. Therefore, from Lemma 2.1 we get that the gossiping time $g(G)$ of any graph with $n$ nodes is always lower-bounded by

$$
g(G) \geq \begin{cases}n-1 & \text { if } n \text { is even, } \\ n & \text { if } n \text { is odd. }\end{cases}
$$

We will show that this lower bound is attained by Hamiltonian graphs.

Let $C_{n}=(V, E)$ denote the ring of length $n$; we assume that the vertex set is $V=\{0, \ldots, n-1\}$ and the edge set is $E=\{(v, w): 1=|v-w|(\bmod n)\}{ }^{1}$

\footnotetext{
${ }^{1}$ Here and in the rest of the paper with $x=a(\bmod b)$ we denote the unique integer $0 \leq x<b$ such that $x=q b+a$.
} 


\section{Gossiping-even $\left(C_{n}\right)$}

Round $t=1$ : Each node $v$ sends its own packet to the node $w$ such that $(v, w) \in M_{1}$.

Round $t=2$ : Each node $v$ sends its own packet to the node $w$ such that $(v, w) \in M_{2}$.

Round $t, 3 \leq t \leq n-1$ : For each node $v$ let $w$ be the node such that $(v, w) \in M_{t}$; node $v$ sends a new packet to $w$; namely, $v$ sends the packet it first received from among those $v$ has neither received from $w$ nor sent to $w$ in any previous round.

FIG. 1. Gossiping algorithm in $C_{n}, n$ even.

\section{Gossiping-odd $\left(C_{n}\right)$}

Round $t, 1 \leq t \leq n$ : For each node $v \neq t-1$ let $w$ be the neighbor of $v$ in $M_{t}$; node $v$ sends to $w$ the packet that $v$ first received from among those that it neither got from $w$ nor sent to $w$ in a previous round ( $v$ 's own packet is considered to be received before any other packet).

FIG. 2. Gossiping algorithm in $C_{n}, n$ odd.

Lemma 2.3. $g\left(C_{n}\right) \leq \begin{cases}n-1 & \text { if } n \text { is even, } \\ n & \text { if } n \text { is odd. }\end{cases}$

Proof. We distinguish two cases according to the parity of the number $n$ of nodes.

Case $n$ even. We shall give a gossiping protocol on the ring $C_{n}$ that requires $n-1$ rounds. First, for each integer $t$, define the perfect matching in $C_{n}$ given by

$$
M_{t}= \begin{cases}\{(v, w): v \text { is even and } w=v+1\} & \text { if } t \text { is even, } \\ \{(v, w): v \text { is odd and } w=v+1(\bmod n)\} & \text { if } t \text { is odd; }\end{cases}
$$

notice that $M_{t}$ and $M_{t+1}$ are disjoint for each $t$. The gossiping algorithm is shown in Figure 1.

It is immediate to see that each node receives a new packet at each round (this can be formally proved by induction on $t$ ). Therefore, at the end of round $n-1$ of algorithm Gossiping-even $\left(C_{n}\right)$ each node has received all the packets of the other $n-1$ nodes.

Case $n$ odd. Define the following maximum matchings $M_{t}$ in $C_{n}$ for each $t=$ $1, \ldots, n$ :

(6)

$M_{t}=\{(v, w): v-t+1(\bmod n)$ is odd, $w=v+1(\bmod n)$, and $v \neq t-1 \neq w\}$.

We give in Figure 2 a gossiping protocol on $C_{n}$ that requires $n$ rounds. It is easy to see that at each round $t=1, \ldots, n$ each node different from $t-1$ receives a new packet. Therefore, at the end of round $n$ of algorithm Gossiping-odd $\left(C_{n}\right)$, each node has received all the packets of the other $n-1$ nodes.

Example 2.1. For $n=6$ we have $M_{1}=M_{3}=M_{5}=\{(1,2),(3,4),(50)\}$ and $M_{2}=M_{4}=\{(0,1),(2,3),(4,5)\}$. Each column of Table 1 shows the set of nodes whose packets are known by $v$ at the end of round $t$, for each $0 \leq v \leq 5$ and $1 \leq t \leq 5$. 


\begin{tabular}{|l|c|c|c|}
\hline$t \backslash v$ & 0 & 1 & 2 \\
\hline 1 & $\{5,0\}$ & $\{1,2\}$ & $\{1,2\}$ \\
2 & $\{5,0,1\}$ & $\{0,1,2\}$ & $\{1,2,3\}$ \\
3 & $\{4,5,0,1\}$ & $\{0,1,2,3\}$ & $\{0,1,2,3\}$ \\
4 & $\{4,5,0,1,2\}$ & $\{5,0,1,2,3\}$ & $\{0,1,2,3,4\}$ \\
5 & $\{3,4,5,0,1,2\}$ & $\{5,0,1,2,3,4\}$ & $\{5,0,1,2,3,4\}$ \\
\hline
\end{tabular}

\begin{tabular}{|l|c|c|c|}
\hline$t \backslash v$ & 3 & 4 & 5 \\
\hline 1 & $\{3,4\}$ & $\{3,4\}$ & $\{5,0\}$ \\
2 & $\{2,3,4\}$ & $\{3,4,5\}$ & $\{4,5,0\}$ \\
3 & $\{2,3,4,5\}$ & $\{2,3,4,5\}$ & $\{4,5,0,1\}$ \\
4 & $\{1,2,3,4,5\}$ & $\{2,3,4,5,0\}$ & $\{3,4,5,0,1\}$ \\
5 & $\{1,2,3,4,5,0\}$ & $\{1,2,3,4,5,0\}$ & $\{3,4,5,0,1,2\}$ \\
\hline
\end{tabular}

TABLE 2

\begin{tabular}{|l|c|c|c|c|c|}
\hline$t \backslash v$ & 0 & 1 & 2 & 3 & 4 \\
\hline 1 & $\{0\}$ & $\{1,2\}$ & $\{1,2\}$ & $\{3,4\}$ & $\{3,4\}$ \\
2 & $\{4,0\}$ & $\{1,2\}$ & $\{1,2,3\}$ & $\{2,3,4\}$ & $\{3,4,0\}$ \\
3 & $\{4,0,1\}$ & $\{0,1,2\}$ & $\{1,2,3\}$ & $\{2,3,4,0\}$ & $\{2,3,4,0\}$ \\
4 & $\{3,4,0,1\}$ & $\{0,1,2,3\}$ & $\{0,1,2,3\}$ & $\{2,3,4,0\}$ & $\{2,3,4,0,1\}$ \\
5 & $\{3,4,0,1,2\}$ & $\{0,1,2,3,4\}$ & $\{0,1,2,3,4\}$ & $\{1,2,3,4,0\}$ & $\{2,3,4,0,1\}$ \\
\hline
\end{tabular}

For $n=5$ we have $M_{1}=\{(1,2),(3,4)\}, M_{2}=\{(2,3),(4,0)\}, M_{3}=\{(3,4),(0,1)\}$ $M_{4}=\{(4,0),(1,2)\}$, and $M_{5}=\{(0,1),(2,3)\}$. Each column of Table 2 shows the set of nodes whose packets are known by $v$ at the end of round $t$, for each $0 \leq v \leq 4$ and $1 \leq t \leq 5$.

THEOREM 2.1. For any Hamiltonian graph $G$ on $n$ vertices,

$$
g(G)= \begin{cases}n-1 & \text { if } n \text { is even } \\ n & \text { if } n \text { is odd. }\end{cases}
$$

Proof. If $G$ is Hamiltonian, from Lemma 2.3 we get that gossiping along the edges of the Hamiltonian cycle requires time matching the lower bound (4), and the theorem holds.

2.3. Trees. In this section we investigate the gossiping time in trees. We first give an upper bound on the gossiping time in any tree and afterwards compute the exact gossiping time of rooted $k$-ary trees.

Consider a tree $T=(V, E)$. We recall that for each node $v$ the set $V_{1}(v)$ denotes the largest of the connected components into which $T$ splits after the removal of $v$. Let

$$
\vartheta=\max \left|V_{1}(v)\right|,
$$

where the maximum is taken over all the internal nodes $v$ having exactly $\operatorname{deg}(v)-1$ leaves as neighbors; notice that any internal node $u$ with less than $d(u)-1$ leaves as neighbors has $\left|V_{1}(u)\right| \leq \vartheta-1$.

Let pre-leaf denote a node $v$ such that $\left|V_{1}(v)\right|=\vartheta$ and denote by $\pi$ the maximum degree of a node in the subgraph consisting only of the edges $(u, f)$ where $f$ is either a leaf or a pre-leaf of $T$. Finally, let $\lambda$ be the maximum number of leaves connected to a common node and $\Delta=\max _{v \in V} \operatorname{deg}(v)$. 
Gossiping-tree $(T)$

Phase 1

[Color each edge $(u, v)$ of $T$ with color $c(u, v)=c(v, u) \in\{0, \ldots, \Delta-1\}$.]

Round $t$, for $t=1, \ldots, \Delta(\vartheta-1)$ : For each node $u$, if there is an edge $(u, v)$ such that $c(u, v)=t-1(\bmod \Delta)$, then $u$ sends a new packet to $v$; namely, $u$ sends to $v$ a packet from among those that $u$ has neither sent to $v$ nor received Phase 2 from $v$ in a previous round, if such a packet exists; otherwise $u$ sends nothing.

[Give to each edge $(u, f)$, where $f$ is a leaf or a pre-leaf, a color $c^{\prime}(u, f) \in$ $\{0, \ldots, \pi-1\}$.]

Round $\Delta(\vartheta-1)+t$, for $t=1, \ldots, \pi$ : For each leaf or pre-leaf $f$, if there is an edge $(u, f)$ with $c^{\prime}(u, f)=t-1$, then $u$ sends to $f$ a packet from among those that $u$ has neither sent to $f$ nor received from $f$ in a previous round, if any.

Phase 3

[Give to each edge $(u, f)$, where $f$ is a leaf of $T$, a color $c^{\prime \prime}(u, f) \in\{0, \ldots, \lambda-$ 1\}.]

Round $\Delta(\vartheta-1)+\pi+t$, for $t=1, \ldots,(n-\vartheta-1) \lambda$ : For each leaf $f$, if the edge $(u, f)$ on $f$ has $c^{\prime \prime}(u, f)=t-1(\bmod \lambda)$, then $u$ sends to $f$ any packet $f$ does not know.

FIG. 3. Gossiping algorithm in a tree $T$.

Theorem 2.2. For any tree $T=(V, E)$ on $n$ nodes, $g(T) \leq(\vartheta-1) \Delta+\pi+(n-$ $\vartheta-1) \lambda$.

Proof. Consider the gossiping algorithm Gossiping-tree $(T)$ given in Figure 3. For any $e \in E$, the edge coloring $c(e)$ and the partial edge colorings $c^{\prime}(e)$ and $c^{\prime \prime}(e)$ used in Gossiping-tree $(T)$ are each intended so that no two edges sharing a vertex are assigned the same color.

We now prove the correctness of algorithm Gossiping-tree(T). Let us say that the edge $(u, v)$ of $T$ is saturated from $u$ to $v$ at time $t$ of Gossiping-tree $(T)$ if no packet is sent from $u$ to $v$ at any time $t^{\prime} \geq t$, that is, if by time $t-1$ node $v$ has received the packet of each node $w$ connected to $v$ through $u$. We need the following property of Gossiping-tree $(T)$.

PROPERTY 2.1. In any round $t$ of Phase 1 of the algorithm Gossiping-tree(T), if the edge $(u, v)$ has color $c(u, v)=t-1(\bmod \Delta)$ and it is not saturated from $u$ to $v$ at time $t$, then $u$ sends a new packet to $v$ at round $t$.

Proof. The proof is by induction on the time unit $t$. Let $t \leq \Delta$ : at time unit $t$, for each edge $(u, v)$ of color $c(u, v)=t-1 \in\{0, \ldots, \Delta-1\}$, nodes $u$ and $v$ exchange a call for the first time and have at least their own packet to send each other.

Now let $t>\Delta$ and suppose that the hypothesis holds for each $t^{\prime}<t$.

Consider an edge $(u, v)$ such that $c(u, v)=t-1(\bmod \Delta)$. Suppose by contradiction that at time $t$ the edge $(u, v)$ is not saturated from $u$ to $v$, but $u$ has no packets to send to $v$ among those $u$ has not received through $v$. That is, all packets known to $u$ and not received through $v$ have already been sent from $u$ to $v$.

In particular, node $u$ has already sent to $v$ all the packets it has received from its other neighbors, call them $w_{1}, \ldots, w_{k}$. Notice that the last call from $u$ to $v$ has taken place at time $t-\Delta$. For each $w_{i}$, let $\tau_{i}$ be the only integer such that both 
$t-\Delta<\tau_{i}<t$ and $c\left(u, w_{i}\right)=\tau_{i}-1(\bmod \Delta)$ hold. If the edge $\left(u, w_{i}\right)$ is not saturated at time $\tau_{i}$, we know by the inductive hypothesis that $u$ has received a packet from $w_{i}$ at time $\tau_{i}$. We can have two cases: the first case is that all the edges $\left(u, w_{i}\right)$ are saturated at time $\tau_{i}<t$. This immediately implies that $(u, v)$ is saturated at time $t$, contradicting our assumption that $(u, v)$ was not saturated from $u$ to $v$ at time $t$. The second case is that at least one edge $\left(u, w_{i}\right)$ is not saturated at time $\tau_{i}$; in such a situation we know by the inductive hypothesis that $u$ has received a new packet from $w_{i}$ at time $\tau_{i}$ that can now be forwarded to $v$, again getting a contradiction.

We can now complete the proof of the theorem by showing that at the end of Gossiping-tree $(T)$ each node knows all the other $n-1$ packets. Property 2.1 shows that a new packet is sent from $u$ to $v$ at each round $t$ of Phase 1 such that $c(u, v)=$ $t-1(\bmod \Delta)$, until the edge $(u, v)$ is saturated and no more packets need to be sent from $u$ to $v$. Therefore, for any internal node $u$ and for any $\Delta$ consecutive rounds, $u$ receives a new packet from each neighbor $v$ such that $(v, u)$ is not saturated from $v$ to $u$. We recall that $\vartheta$ is the maximum number of packets that any internal node needs to get from one neighbor and that this maximum is attained with equality only if $u$ is a pre-leaf. Therefore, by round $\Delta(\vartheta-1)$, any node $u$ which is not a pre-leaf gets all the necessary $n-1$ packets, while a pre-leaf gets $n-2$ packets during the $\Delta(\vartheta-1)$ rounds of Phase 1, and the remaining packet during some round of Phase 2.

Analogously, during Phase 1 any leaf $f$ gets $\vartheta-1$ packets. It is obvious that $f$ receives a new packet during Phase 2; moreover, during Phase 3 the leaf $f$ receives a new packet for any $\lambda$ consecutive rounds, thus getting the remaining $n-\vartheta-1$ packets that it needs to complete the gossip.

Let $\delta$ denote the minimum degree of an internal node in $T$. It is easy to see that we can upper-bound $\vartheta$ by $n-\delta$. Therefore, from Theorem 2.2 we have the following upper bound on $g(T)$ that is expressed only in terms of degree properties of the nodes in $T$.

Corollary 2.3. For any tree $T=(V, E)$ on $n$ nodes, $g(T) \leq(n-\delta) \Delta+(\delta-1) \lambda$.

Given a connected graph $G=(V, E)$, denote by $\mathcal{T}$ the set of all spanning trees of $G$, and for any vertex $v \in V$, denote by $\operatorname{deg}_{T}(v)$ the degree of $v$ in $T \in \mathcal{T}$. Define $d(G)=\min _{T \in \mathcal{T}} \max _{v \in V} \operatorname{deg}_{T}(v)$. The following corollary is immediate.

Corollary 2.4. For any connected graph $G=(V, E)$ with $n$ vertices,

$$
g(G) \leq(n-1) d(G) .
$$

We point out that, although the problem of computing $d(G)$ is NP-hard, there exists an efficient algorithm to compute a spanning tree of maximum degree at most $d(G)+1$ (see [12]). From Corollaries 2.2 and 2.4 we have that for any tree with $n$ nodes and maximum degree $\Delta$ it holds that $n \Delta-n+1 \leq g(T) \leq n \Delta-\Delta$. Let us now consider the tree $S_{n, \Delta}$ of Figure 4. If $\Delta=n-1$, then $S_{n, n-1}$ is the star on $n$ nodes, and from Corollary 2.2 and Theorem 2.2 we have $g\left(S_{n, n-1}\right)=(n-1)^{2}$. If $\Delta>2$ is constant with respect to $n>2 \Delta$ then from Corollary 2.2 and Theorem 2.2 we get $\Delta(n-1)-(\Delta-1) \leq g\left(S_{n, \Delta}\right) \leq \Delta(n-1)-2$. It is not difficult to obtain a specific gossiping algorithm attaining the lower bound, hence the bound (7) is asymptotically tight.

In [7] it was conjectured that for any $G, g_{H_{1}}(1, G)=\Omega(n d(G))$ holds; Ravi [22] has proved the following theorem.

Theorem 2.3 (see [22]). For any graph $G, g(G)=\Theta(n d(G))$. 


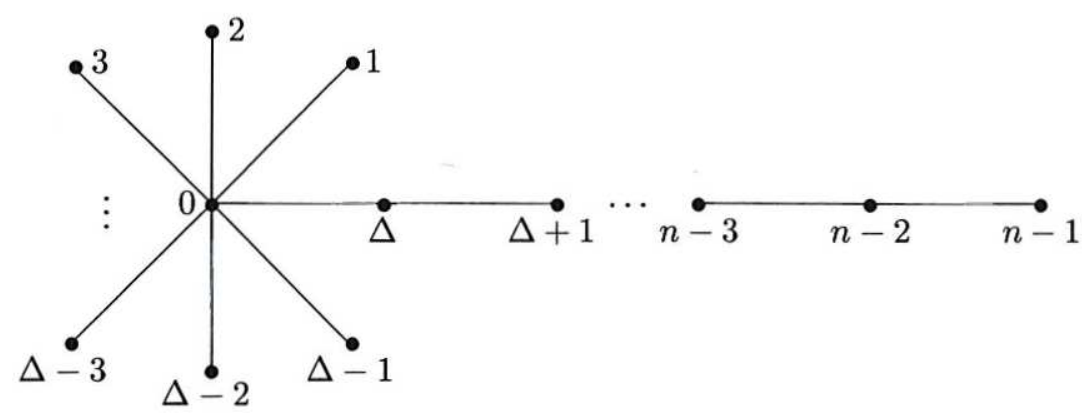

FIG. 4. Tree $S_{n, \Delta}$.

Proof. Given a vertex cutset $X$ of $G=(V, E)$, from Lemma 2.2 we know that

$$
g(G) \geq\left\lceil\sum_{i=1}^{c(X)} \frac{\max \left\{\left|V_{i}\right|, n-\left|V_{i}\right|\right\}}{\left|M_{X}\right|}\right\rceil,
$$

where $V_{1}, \ldots, V_{c(X)}$ are the connected components induced in $G$ by $V-X$ and $M_{X}$ is a maximum matching between $X$ and $V-X$ in $G$. Noticing that $\left|M_{X}\right| \leq|X|$ and $\max \left\{\left|V_{i}\right|, n-\left|V_{i}\right|\right\} \geq n / 2$ for each $i$,

$$
g(G) \geq \frac{n}{2} \frac{c(X)}{|X|} .
$$

It was proved by Fürer and Raghavachari (see [12, Section 5]) that there exists a vertex cutset $Y \subset V$ such that

$$
d(G) \leq \frac{c(Y)}{|Y|}+1
$$

Using the above inequality and the lower bound (8), we have

$$
g(G) \geq \frac{n}{2}(d(G)-1) .
$$

The above inequality, together with Corollary 2.4, implies that $g(G)=\Theta(n d(G))$. 口

We remark that the same reasoning as in Theorem 2.3 allows us to prove that for any $p$

$$
g_{F_{1}}(p, G)=\Omega(n d(G) / p) .
$$

We shall now compute the exact gossiping time of $k$-ary trees, that is, rooted trees in which each internal node has exactly $k$ sons. Let $T_{k, n}$ denote any $k$-ary tree with $n$ nodes.

Let us first notice that for $n=k+1$ the tree $T_{k, n}$ is the star $S_{k+1, k}$. Consider then a tree $T_{k, n}$ with $n \geq 2 k+1$ nodes. Let $u$ be a node of $T_{k, n}$ whose sons are all leaves. By Corollary 2.2 we get

$$
g\left(T_{k, n}\right) \geq \max _{v} L(v) \geq L(u)= \begin{cases}k n+1 & \text { if } n=2 k+1, \\ (k+1)(n-1)-k & \text { if } n \geq 3 k+1 .\end{cases}
$$




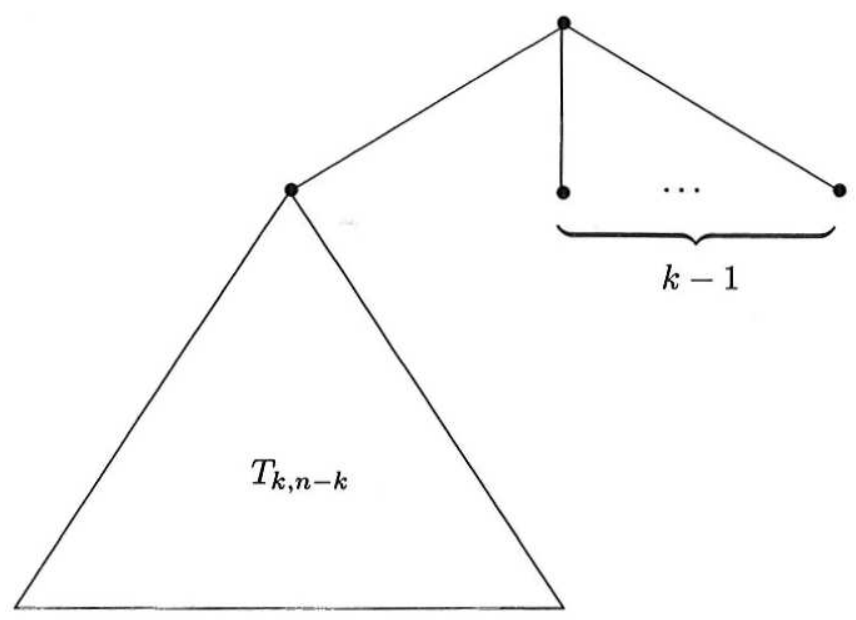

FIG. 5.

We show now that (9) holds with equality. Applying Theorem 2.2 to $T_{k, n}$ we get that

$$
g\left(T_{k, n}\right) \leq(\vartheta-1) \Delta+\pi+(n-\vartheta-1) \lambda=(\vartheta-1)(k+1)+\pi+(n-\vartheta-1) k .
$$

Unless exactly $k-1$ sons of the root are leaves (cf. the tree in Figure 5), $T_{k, n}$ has $\vartheta=n-k-1$ and $\pi \leq \Delta=k+1$; that, by (10) and (9), gives

$$
g\left(T_{k, n}\right)=(n-k-2)(k+1)+k+1+k^{2}=(k+1)(n-1)-k .
$$

Consider the remaining case when $T_{k, n}$ is the tree of Figure 5 . The only pre-leaf is the root, and $\vartheta=n-k$. If $n \geq 3 k+1$ we have $\pi=k$, and from (10) we get

$$
g\left(T_{k, n}\right) \leq(n-k-1)(k+1)+k+(k-1) k=(n-1)(k+1)-k ;
$$

if $n=2 k+1$ we have $\pi=\Delta=k+1$ and $g\left(T_{k, 2 k+1}\right) \leq k n+1$.

Therefore, we have proved the following result.

THEOREM 2.4. For any $k$-ary tree on $n$ nodes $T_{k, n}$,

$$
g\left(T_{k, n}\right)= \begin{cases}k^{2} & \text { if } n=k+1, \\ 2 k^{2}+k+1 & \text { if } n=2 k+1, \\ (k+1)(n-1)-k & \text { if } n \geq 3 k+1 .\end{cases}
$$

The particular case $k=1$ of the above result deserves to be explicitly stated.

Corollary 2.5. Let $P_{n}$ be the path on $n$ nodes. We have

$$
g\left(P_{n}\right)= \begin{cases}1 & \text { if } n=2, \\ 4 & \text { if } n=3, \\ 2 n-3 & \text { if } n \geq 4 .\end{cases}
$$

2.4. Complete bipartite graphs. Let $K_{r, s}=\left(V\left(K_{r, s}\right), E\left(K_{r, s}\right)\right)$ be the complete bipartite graph on the node set $V\left(K_{r, s}\right)=\left\{a_{0}, \ldots, a_{r-1}\right\} \cup\left\{b_{0}, \ldots, b_{s-1}\right\}$, with $r \geq s,\left\{a_{1}, \ldots, a_{r-1}\right\} \cap\left\{b_{0}, \ldots, b_{s-1}\right\}=\emptyset$, and edge set $E\left(K_{r, s}\right)=\left\{a_{0}, \ldots, a_{r-1}\right\} \times$ $\left\{b_{0}, \ldots, b_{s-1}\right\}$. In the next theorem we determine the gossiping time of $K_{r, s}$. 
THEOREM 2.5. For each $r$ and $s$ with $r \geq s \geq 1$, it holds that $g\left(K_{r, s}\right)=$ $\lceil(r+s-1) r / s\rceil$.

Proof. The lower bound $g\left(K_{r, s}\right) \geq\lceil(r+s-1) r / s\rceil$ is an immediate consequence of Corollary 2.1 since the complete bipartite graph has $\alpha\left(K_{r, s}\right)=r$.

In order to give a gossiping algorithm in $K_{r, s}$ requiring $\lceil(r+s-1) r / s\rceil$ communication rounds, we define the matchings

$$
M_{j}=\left\{\left(b_{i}, a_{i+j(\bmod r)}\right): 0 \leq i \leq s-1\right\}
$$

for $j=0, \ldots, r-1$. The algorithm is shown in Figure 6 .

According to the protocol, at the end of Phase 1 of Gossiping-bipartite $\left(K_{r, s}\right)$ each node $a_{i}$ (resp., $b_{i}$ ) knows the message of each $b_{i}$ (resp., $a_{i}$ ). Consider now Phase 2. It is immediate to see that during the first $s-1$ rounds of Phase 2 each of the $b_{i}$ 's receives the packet of each $b_{j}$ for $j \neq i$, thus completing its knowledge. Moreover, after the $\lceil r(r+s-1) / s\rceil-r=\lceil r(r-1) / s\rceil$ rounds of Phase 2 each node $a_{i}$ has been involved in a call at least $r-1$ times and has then received the packet of each of the $a_{j}$, for $j \neq i$, thus completing its knowledge.

\section{Gossiping-bipartite $\left(K_{r, s}\right)$}

Phase 1

round $t$, for $t=1, \ldots, r$ : For each edge $\left(b_{i}, a_{i+t-1(\bmod r)}\right) \in M_{t-1}$

Phase 2 nodes $b_{i}$ and $a_{i+t-1}(\bmod r)$ exchange their own packets.

round $t$, for $t=r+1, \ldots,\lceil r(r+s-1) / s\rceil$ :

For each edge $\left(b_{i}, a_{j}\right) \in M_{(t-1-r) s(\bmod r)}$ node $b_{i}$ sends to $a_{j}$ any packet that $a_{j}$ has not received in a previous round;

if $t \leq r+s-1$ then $b_{i}$ receives from $a_{j}$ the packet of $b_{i+t-r(\bmod s)}$.

FIG. 6. Gossiping algorithm in $K_{r, s}$.

2.5. Generalized Petersen graphs. In section 2.2 .1 we have seen that Hamiltonian graphs have the minimum possible gossiping time among all graphs with $n$ nodes. It is natural to wonder whether there are non-Hamiltonian graphs on $n$ vertices with gossiping time equal to $n$ if $n$ is odd and $n-1$ if $n$ is even. A quick check shows that this is not the case for rectangular grids $G_{t, s}$ with both $t$ and $s$ odd. ${ }^{2}$ In fact, we know that $\alpha\left(G_{t, s}\right)=\left\lceil\frac{s \cdot t}{2}\right\rceil$, and from Corollary 2.1, we get $g\left(G_{t, s}\right) \geq s \cdot t+1$. Moreover, it is also easy to check that the gossiping time of the Petersen graph on 10 vertices is at least 10 . Therefore, one could be tempted to conjecture that the gossiping time $g(G)$ of a graph $G$ is equal to the minimum possible only if $G$ is Hamiltonian. This conjecture, although nice sounding, would be wrong, as the following classes of graphs, including the generalized Petersen graphs (GPGs), show.

Let $P_{k, \pi}$ be the graph consisting of two cycles of size $k$ connected by a perfect matching in the following way: given a permutation $\pi$ of $\{0, \ldots, k-1\}$ the graph $P_{k, \pi}=\left(V\left(P_{k, \pi}\right), E\left(P_{k, \pi}\right)\right)$ has vertex set $V\left(P_{k, \pi}\right)=\left\{a_{0}, \ldots, a_{k-1}\right\} \cup\left\{b_{0}, \ldots, b_{k-1}\right\}$ and edge set

\footnotetext{
${ }^{2}$ It is well known that all rectangular grids $G_{t, s}$ are Hamiltonian except when $t$ and $s$ are both odd.
} 


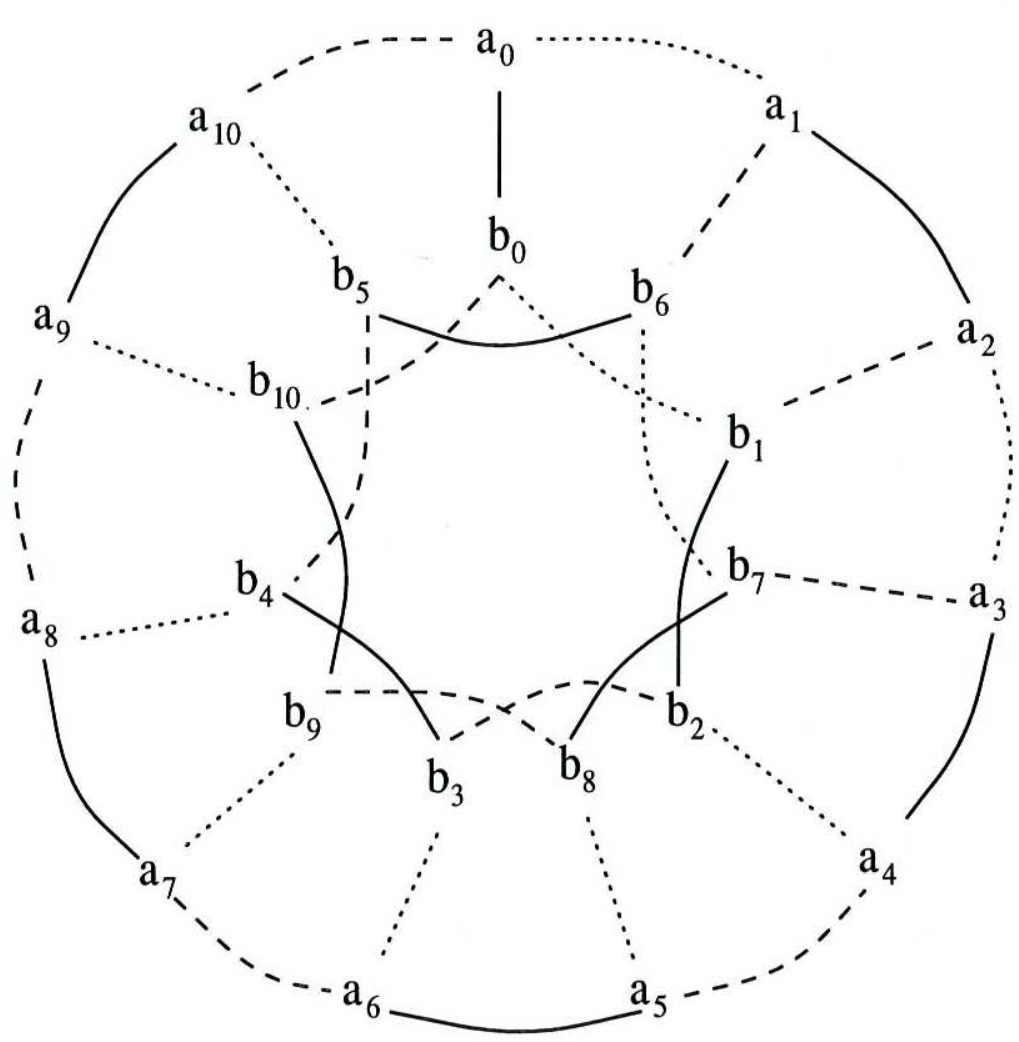

Fig. 7. A 3-coloration of the GPG with $n=11$ and $s=2$.

$$
\begin{aligned}
E\left(P_{k, \pi}\right) & =\left\{\left(a_{i}, a_{i+1(\bmod k)}\right): 0 \leq i<k\right\} \\
& \cup\left\{\left(b_{i}, b_{i+1(\bmod k)}\right): 0 \leq i<k\right\} \cup\left\{\left(a_{i}, b_{\pi(i)}\right): 0 \leq i<k\right\} .
\end{aligned}
$$

The Petersen graph has $k=5$ and $\pi(i)=3 i(\bmod 5)$, for $i=0,1,2,3,4$; GPGs have $k$ odd and $\pi(s \cdot i(\bmod k))=i, i=0, \ldots, k-1$, for a fixed integer $s$.

From Lemma 2.1 we know that $g\left(P_{k, \pi}\right) \geq\left|V\left(P_{k, \pi}\right)\right|-1=2 k-1$. We will show that for any $k$ and $\pi$ such that $P_{k, \pi}$ is 3-edge-colorable, we have the equality

$$
g\left(P_{k, \pi}\right)=2 k-1 .
$$

Each cubic GPG, other than the Petersen graph itself, is 3-edge-colorable [2]. Moreover, the class of 3-edge-colorable $P_{k, \pi}$ 's includes the family of non-Hamiltonian GPGs with $k=5(\bmod 6)$ and $s=2$ (see [2] and references therein quoted). For an example of a 3-coloration of a GPG, see Figure 7.

The gossiping algorithm is described in Figure 8; it assumes that the edges of the graph are colored with the three colors 1,2 , and 3 . It is easy to prove by induction on $q$ that all the calls of Phase $q$, for $q \leq(k-1) / 2$, can actually be done. Therefore, after the first $(k-1) / 2$ phases, each node $a_{i}$ has the packet of $a_{i \pm j(\bmod k)}$ for $j=$ $0, \ldots,(k-1) / 2$; that is, it knows the packet of each other node in its own cycle; moreover, it knows the packet of $(k-1) / 2$ nodes in the cycle on $\left\{b_{0}, \ldots, b_{k-1}\right\}$. Analogously, each $b_{i}$ knows the packet of each other node in its own cycle and of $(k-1) / 2$ nodes in $\left\{a_{0}, \ldots, a_{k-1}\right\}$. 
Gossiping-3-color $\left(P_{k, \pi}\right)$

Phase $q(1 \leq q \leq(k-1) / 2)$ [each phase consists of three communication rounds]: round $t(t=1,2,3)$ : make a call between the endpoints of each edge of color $t$.

Calls are made so that:

when an edge $\left(a_{i}, a_{i+1(\bmod k)}\right)$ is used, then $a_{i}$ receives the packet of $a_{i+q(\bmod k)}$, and $a_{i+1(\bmod k)}$ receives the packet of $a_{i+1-q(\bmod k)}$;

when an edge $\left(b_{i}, b_{i+1(\bmod k)}\right)$ is used, then $b_{i}$ receives the packet of $b_{i+q(\bmod k)}$, and $b_{i+1(\bmod k)}$ receives the packet of $b_{i+1-q(\bmod k)}$;

when the edge $\left(a_{i}, b_{\pi(i)}\right)$ is used, then $a_{i}$ receives the packet of some $b_{j}, 0 \leq$ $j \leq k-1$, and $b_{\pi(i)}$ receives the packet of some $a_{j}, 0 \leq j \leq k-1$.

Phase $3(k-1) / 2+q(1 \leq q \leq(k+1) / 2)$ the phase consists of one communication round]:

node $a_{i}$ (resp., $\left.b_{\pi(i)}\right)$, for $i=0, \ldots, k-1$, sends to $b_{\pi(i)}$ (resp., $a_{i}$ ) the packet of some $a_{j}$ (resp., $b_{j}$ ) it has not already sent to it.

FIG. 8. Gossiping algorithm in $P_{k, \pi}$.

Therefore, the calls between nodes in $\left\{a_{0}, \ldots, a_{k-1}\right\}$ and in $\left\{b_{0}, \ldots, b_{k-1}\right\}$ of the last $(k+1) / 2$ communication rounds allow completion of the knowledge of each node in the graph.

3. Gossiping by exchanging more than one packet at a time. In this section we shall study the minimum number of time units $g_{F_{1}}(p, G)$ necessary to perform gossiping in a graph $G$, under the restriction that at each time instant communicating nodes can exchange up to $p$ packets, $p$ fixed but arbitrary otherwise. We assume that $p$ is smaller than the number of nodes of the graph $G$; otherwise, the problem is equivalent to the classical one. Again, for ease of notation, we shall write $g(p, G)$ to denote $g_{F_{1}}(p, G)$.

3.1. Lower bounds. First, we shall present a simple lower bound on $g(p, G)$ based on elementary counting arguments. Nonetheless, we shall prove in the sequel that the obtained lower bound is tight for complete graphs with an even number of nodes and for hypercubes. In order to derive the lower bound, let us define $I(p, t)$ as the maximum number of packets a vertex can have possibly received after $t$ communication rounds in any graph. Since at each round $i$, with $1 \leq i \leq t$, any vertex can receive at $\operatorname{most} \min \left\{p, 2^{i-1}\right\}$ packets, it follows that

$$
I(p, t)=1+\sum_{i=1}^{t} \min \left\{p, 2^{i-1}\right\}
$$

or equivalently,

$$
I(p, t)=1+\sum_{i=1}^{\lceil\log p\rceil} 2^{i-1}+p(t-\lceil\log p\rceil)=2^{\lceil\log p\rceil}+p(t-\lceil\log p\rceil)
$$

for any $t \geq\lceil\log p\rceil$. Therefore, for any graph $G=(V, E)$, the gossiping time $g(p, G)$ is always lower bounded by the smallest integer $t^{\star}$ for which $I\left(p, t^{\star}\right) \geq|V|$. Since $t^{\star}$ 
is obviously greater than or equal to $\lceil\log |V|\rceil \geq\lceil\log p\rceil$, we can use (12) and obtain

$$
g(p, G) \geq\lceil\log p\rceil+\left\lceil\frac{1}{p}\left(|V|-2^{\lceil\log p\rceil}\right)\right\rceil .
$$

Moreover, notice that if the number of nodes in the graph is odd, then at each round there is a node that does not receive any message. This implies that after any round $t$ there exists a node that can have possibly received at most $I(p, t-1)$ packets. Therefore,

$$
g(p, G) \geq\lceil\log p\rceil+\left\lceil\frac{1}{p}\left(|V|-2^{\lceil\log p\rceil}\right)\right\rceil+1 .
$$

The above arguments give the following lemma.

Lemma 3.1. For any graph $G=(V, E),|V|=n$, and integer $p$ such that $2^{\lceil\log p\rceil} \leq$ $n$, we have

$$
g(p, G) \geq \begin{cases}\lceil\log p\rceil+\left\lceil\frac{1}{p}\left(n-2^{\lceil\log p\rceil}\right)\right\rceil & \text { if } n \text { is even } \\ \lceil\log p\rceil+\left\lceil\frac{1}{p}\left(n-2^{\lceil\log p\rceil}\right)\right\rceil+1 & \text { if } n \text { is odd }\end{cases}
$$

Using similar arguments, we can also generalize the lower bound (1) that we established in section 2.1 for $p=1$ to general values of $p$.

Lemma 3.2. Let $G=(V, E)$ be a graph with $n$ vertices and let $\mu(G)$ be the size of a maximum matching in $G$. For any integer $p$ such that $2^{[\log p\rceil} \leq n$,

$$
g(p, G) \geq\lceil\log p\rceil+\left\lceil\frac{1}{p}\left(\frac{n(n-1)}{2 \mu(G)}-2^{\lceil\log p\rceil}+1\right)\right\rceil .
$$

Remark 3.1. Given a gossiping algorithm $\mathcal{A}$ for a graph $G$ that uses messages of size not larger than $p$, we can easily derive from it an algorithm $\mathcal{B}$ to gossip in $G$ with messages of size $q<p$. Indeed, if a message of size $>q$ is sent during a call of $\mathcal{A}$, then we can split this call into more calls, each transmitting up to $q$ packets. For example, we can use this observation to derive the following bound:

(13) $g(p, G) \leq\lfloor\log p\rfloor+1+2(g(2 p, G)-\lfloor\log p\rfloor-1)=2 g(2 p, G)-\lfloor\log p\rfloor-1$.

Bound (13) can be proved by noticing that during the first $\lfloor\log p\rfloor+1$ calls of the algorithm attaining $g(2 p, G)$, the exchanged messages necessarily have size less than or equal to $p$. From this observation and Theorem 2.5, it follows, for example, that for the complete bipartite graph $g\left(2, K_{r, s}\right) \geq\left(g\left(1, K_{r, s}\right)+1\right) / 2=\lceil(r+s-1) r /(2 s)\rceil+1$; it is not difficult to derive an algorithm similar to the one in Figure 6 attaining the equality.

3.2. Rings and paths. Let $g(\infty, G)$ denote the gossiping time of the graph $G$ in the absence of any restriction on the size of the messages. It is obvious that for each $p, g(p, G) \geq g(\infty, G)$ holds; it is possible to see that equality holds for any $p \geq 2$ when $G$ is either the ring $C_{n}$ or the path $P_{n}$ on $n$ nodes.

It is well known that [17]

$$
g\left(\infty, P_{n}\right)=2\left\lceil\frac{n}{2}\right\rceil-1 \quad \text { and } \quad g\left(\infty, C_{n}\right)= \begin{cases}n / 2 & \text { if } n \text { is even, } \\ (n+3) / 2 & \text { if } n \text { is odd }\end{cases}
$$


We just point out that it is easy to see that the algorithms attaining $g\left(\infty, C_{n}\right)$ and $g\left(\infty, P_{n}\right)$ do not need to send more than two packets at a time. Therefore, the following results hold.

THEOREM 3.1. For each $n \geq 3$ and $p \geq 2$,

$$
g\left(p, C_{n}\right)=g\left(2, C_{n}\right)= \begin{cases}n / 2 & \text { if } n \text { is even }, \\ (n+3) / 2 & \text { if } n \text { is odd. }\end{cases}
$$

THEOREM 3.2. For each $n \geq 2$ and $p \geq 2$,

$$
g\left(p, P_{n}\right)=g\left(2, P_{n}\right)=2\left\lceil\frac{n}{2}\right\rceil-1 .
$$

3.3. Complete graphs. In this section we study the gossiping time of the complete graph $K_{n}$ on $n$ nodes. We shall denote by $\{0,1, \ldots, n-1\}$ the vertex set of $K_{n}$. We recall that $g\left(\infty, K_{n}\right)$ is equal to $\lceil\log n\rceil$ if $n$ is even, and $\lceil\log n\rceil+1$ if $n$ is odd.

THEOREM 3.3. For each even integer $n$ and integer $p$ such that $2^{\lceil\log p\rceil} \leq n$,

$$
g\left(p, K_{n}\right)=\lceil\log p\rceil+\left\lceil\frac{n-2^{\lceil\log p\rceil}}{p}\right\rceil .
$$

Proof. The lower bound follows from Lemma 3.1. We now give a gossiping algorithm for $K_{n}$ that uses the optimal number of rounds. For each node $v$, with $v$ even and $0 \leq v \leq n-1$, define the sequence of nodes $v_{t}$ as

$$
v_{t}= \begin{cases}v+2^{t}-1(\bmod n) & \text { if } 1 \leq t \leq\lceil\log p\rceil, \\ v+2^{\lceil\log p\rceil}-1+\tau p+1(\bmod n) & \text { if } t=\lceil\log p\rceil+\tau, \text { with } \tau \geq 1 \text { and } p \cdot \tau \text { odd, } \\ v+2^{\lceil\log p\rceil}-1+\tau p(\bmod n) & \text { if } t=\lceil\log p\rceil+\tau, \text { with either } p \text { or } \tau \geq 1 \text { even. }\end{cases}
$$

Note that for each $t$ the set $M_{t}=\left\{\left(v, v_{t}\right): v\right.$ even, $\left.0 \leq v<n\right\}$ is a perfect matching between even and odd nodes. Finally, for each integer $\tau \geq 1$, for each even node $v$, with $0 \leq v \leq n-1$, define

$$
P_{\text {even }}(n)= \begin{cases}\{v+i(\bmod n): 1 \leq i \leq p\} & \text { if } p \text { and } \tau \text { are odd } \\ \{v+i(\bmod n): 0 \leq i \leq p-1\} & \text { otherwise }\end{cases}
$$

and for each odd node $v$, with $0 \leq v \leq n-1$,

$$
P_{\text {odd }}(v, \tau)= \begin{cases}\{v-i(\bmod n): 1 \leq i \leq p\} & \text { if } p \text { and } \tau \text { are odd } \\ \{v-i(\bmod n): 0 \leq i \leq p-1\} & \text { otherwise. }\end{cases}
$$

Consider the gossiping algorithm given in Figure 9 and let $I_{n}(v, t)$ denote the set of nodes whose packets are known by $v$ by the end of round $t$. For each node $v$ the size of $I_{n}(v, t)$ doubles at each round of Phase 1 and increases by $p$ in every round of Phase 2. Indeed, it is immediate to see that for each $t=1, \ldots,\lceil\log p\rceil$

$$
I_{n}(v, t)= \begin{cases}\left\{v+i(\bmod n): 0 \leq i \leq 2^{t}-1\right\} & \text { if } v \text { is even } \\ \left\{v-i(\bmod n): 0 \leq i \leq 2^{t}-1\right\} & \text { if } v \text { is odd }\end{cases}
$$


Gossiping-even $\left(p, K_{n}\right)$

Phase 1

Round $t, 1 \leq t \leq\lceil\log p\rceil$ : For each even node $v$ nodes $v$ and $v_{t}$ exchange all the packets they know.

Phase 2

Round $t=\lceil\log p\rceil+\tau, 1 \leq \tau \leq\left\lceil\frac{n-2^{\lceil\log p\rceil}}{p}\right\rceil$ : For each even node $v$ node $v$ sends to $v_{t}$ the packets of nodes in $P_{\text {even }}(v, \tau)$ and node $v_{t}$ sends to $v$ the packets of nodes in $P_{\text {odd }}\left(v_{t}, \tau\right)$.

FIG. 9. Gossiping algorithm in $K_{n}, n$ even.

and for each $\tau=1, \ldots,\left\lceil\frac{n-2^{\lceil\log p\rceil}}{p}\right\rceil$

$$
I_{n}(v,\lceil\log p\rceil+\tau)= \begin{cases}\left\{v+i(\bmod n): 0 \leq i \leq 2^{\lceil\log p\rceil}+\tau p-1\right\} & \text { if } v \text { is even } \\ \left\{v-i(\bmod n): 0 \leq i \leq 2^{\lceil\log p\rceil}+\tau p-1\right\} & \text { if } v \text { is odd. }\end{cases}
$$

Hence, $I_{n}\left(v,\lceil\log p\rceil+\left\lceil\frac{n-2^{\lceil\log p\rceil}}{p}\right\rceil\right)=\{0, \ldots, n-1\}=V$ for each node $v$.

Remark 3.2. A close look at the algorithm Gossiping-even $\left(p, K_{n}\right)$ reveals that the calls are always made between even and odd nodes. Therefore, the same protocol works in the complete bipartite graphs $K_{r, r}$ from which we get that for any $p$ and $r$

$$
g\left(p, K_{r, r}\right)=g\left(p, K_{2 r}\right)=\lceil\log p\rceil+\left\lceil\frac{1}{p}\left(2 r-2^{\lceil\log p\rceil}\right)\right\rceil .
$$

We now consider the case of complete graphs with odd number of nodes.

THEOREM 3.4. For each odd integer $N$ and integer $p$ such that $2^{\lceil\log p\rceil} \leq N+1$,

$$
\lceil\log p\rceil+\left\lceil\frac{N-2^{\lceil\log p\rceil}}{p}\right\rceil+1 \leq g\left(p, K_{N}\right) \leq\lceil\log p\rceil+\left\lceil\frac{N+1-2^{\lceil\log p\rceil}}{p}\right\rceil+2 .
$$

Proof. The lower bound follows from Lemma 3.1. To prove the upper bound, we show that the algorithm Gossiping-odd $\left(p, K_{N}\right)$ given in Figure 10 completes gossiping in $K_{N}$ in $\lceil\log p\rceil+\left\lceil\frac{N+1-2^{\lceil\log p\rceil}}{p}\right\rceil+2$ rounds. The algorithm Gossiping$\operatorname{odd}\left(p, K_{N}\right)$ is described in terms of the algorithm $\operatorname{Gossiping-even}\left(p, K_{n}\right)$, where $n=N+1$.

Let $V_{t}, P_{\text {even }}(v, \tau)$, and $P_{\text {odd }}(v, \tau)$ be defined as in (14), (15), and (16), respectively. In order to show the correctness of Gossiping-odd $\left(p, K_{N}\right)$, let us first consider Phase 1. At round $t$, for $1 \leq t \leq\lceil\log p\rceil$, node $N+1-2^{t}$ does not receive the information of the nodes in $I_{n}(N, t)-\{N\}$. It is easy to see that the nodes that do not have the packets of all the nodes in $I_{n}(v, t)$ are the nodes in the set $X_{t}$ defined by $X_{1}=\emptyset$, and

$$
\begin{aligned}
X_{t}=X_{t-1} \cup\left\{v+2^{t}-1(\bmod n):\right. & \left.: v \in X_{t-1} \text { even }\right\} \\
\cup\left\{v-2^{t}+1(\bmod n):\right. & \left.: v \in X_{t-1} \text { odd }\right\} \cup\left\{N+1-2^{t}\right\}
\end{aligned}
$$


Gossiping-odd $\left(p, K_{N}\right)$

Phase 1

Round $t, 1 \leq t \leq\lceil\log p\rceil$ : For each even node $v$, with $v \neq N+1-2^{t}$, nodes $v$ and $v_{t}$ exchange all the packets they know;

Round $t=\lceil\log p\rceil+1$ : each node $v$ with

$v \in\left\{3+4 i: 0 \leq i \leq 2^{\lceil\log p\rceil-2}-2\right\} \cup\left\{N-3-4 i: 0 \leq i \leq 2^{\lceil\log p\rceil-2}-1\right\}$ receives from $v+2$ a message containing the packets of all the nodes in $\{N-$ Phase 2 $\left.2^{\lceil\log p\rceil-1}+1, \ldots, N-1\right\}$.

Round $t=\lceil\log p\rceil+1+\tau, 1 \leq \tau \leq\left\lceil\left(N+1-2^{\lceil\log p\rceil}\right) / p\right\rceil$ : For each even $v$ with $v_{t-1} \neq N$ node $v$ sends to $v_{t-1}$ the packets of nodes in $P_{\text {even }}(v, \tau)$ and $v_{t-1}$ sends to $v$ the packets of nodes in $P_{\text {odd }}\left(v_{t-1}, \tau\right)$.

Round $t=\lceil\log p\rceil+\left\lceil\left(N+1-2^{\lceil\log p\rceil}\right) / p\right\rceil+2$ : Each node $v$ such that $v_{t-1}=$ $n-1$ for some $t=\lceil\log p\rceil+1+\tau$ with $1 \leq \tau \leq\left\lceil\left(N+1-2^{\lceil\log p\rceil}\right) / p\right\rceil+1$ receives from $v+1$ a message containing the packets of the nodes in $P_{\text {odd }}(N, \tau)$.

FIG. 10. Gossiping algorithm in $K_{N}, N$ odd.

for $2 \leq t \leq\lceil\log p\rceil$. This gives

$$
X_{t}=\left\{3+4 i: 0 \leq i \leq 2^{t-2}-2\right\} \cup\left\{N-3-4 i: 0 \leq i \leq 2^{t-2}-1\right\}
$$

for $t=2, \ldots,\lceil\log p\rceil$.

Moreover, each node in $X_{t}$ has at least those packets of all nodes in $I(v, t)-$ $I(N, t-1)$. Therefore, at the end of round $\lceil\log p\rceil$, each node in $X_{\lceil\log p\rceil}$ lacks at most the packets of the nodes in $I(N,\lceil\log p\rceil-1)=\left\{N-2^{\lceil\log p\rceil-1}+1, N-2^{\lceil\log p\rceil-1}+\right.$ $2, \ldots, N-1\}$, and the calls of round $\lceil\log p\rceil+1$ between each node $v \in X_{\lceil\log p\rceil}$ and $v+2 \notin X_{\lceil\log p\rceil}$ assure that each node knows the packets of all nodes in $I(v,\lceil\log p\rceil)$.

Now consider Phase 2. It is immediate that at round $t$ each node receives $p$ new packets, except for the even node $v$ such that $v_{t-1}=N$. Hence, after the calls of round $\lceil\log p\rceil+\left\lceil\frac{n-2^{\lceil\log p\rceil}}{p}\right\rceil+2$, each node knows the packet of each of the other $N-1$ nodes.

For $N$ odd, we believe that the true value of $g\left(p, K_{N}\right)$ is $\lceil\log p\rceil+\left\lceil\frac{N-2^{\lceil\log p\rceil}}{p}\right\rceil+1$; we can verify this equality for small values of $N$ and $p$. In case $p=2$, Theorem 3.1 and Lemma 3.1 tell us that $g\left(2, K_{N}\right)=(N+3) / 2=g\left(2, C_{N}\right)$, for each odd $N \geq 2$. Moreover, we can prove the following theorem.

THEOREM 3.5. If $p$ is a multiple of 4 then $g\left(p, K_{N}\right)=\lceil\log p\rceil+\left\lceil\frac{N-2^{\lceil\log p\rceil}}{p}\right\rceil+1$.

Proof. Execute the first $\lceil\log p\rceil$ rounds of $\operatorname{Gossiping-odd}\left(p, K_{N}\right)$ : from (19) we know that the nodes that have not received the packets of all nodes in $I_{n}(v,\lceil\log p\rceil)$ are those in the set

$$
X_{\lceil\log p\rceil}=\left\{3+4 i: 0 \leq i \leq 2^{\lceil\log p\rceil-2}-2\right\} \cup\left\{N-3-4 i: 0 \leq i \leq 2^{\lceil\log p\rceil-2}-1\right\} .
$$

Continue the gossiping process as follows.

Round $t=\lceil\log p\rceil+\tau, 1 \leq \tau \leq\left\lceil\left(N-2^{\lceil\log p\rceil}\right) / p\right\rceil-1$ : For each even $v$ with $v_{t} \neq N, v$ sends to $v_{t}$ the packets of nodes in $P_{\text {even }}(v, \tau)$ and $v_{t}$ sends to $v$ the packets of nodes in $P_{\text {odd }}\left(v_{t}, \tau\right)$. 
The set $X_{\lceil\log p\rceil+\tau}$ of the nodes that at round $\lceil\log p\rceil+\tau$ do not have the packets of all nodes in $I_{n}(v,\lceil\log p\rceil+\tau)$ satisfies

$$
X_{\lceil\log p\rceil+\tau}=X_{\lceil\log p\rceil+\tau-1} \cup\left\{v: v_{t} \in X_{\lceil\log p\rceil} \cup\{N\}\right\} .
$$

We can then deduce that for each $\tau \leq\left\lceil\left(N-2^{\lceil\log p\rceil}\right) / p\right\rceil-1$,

$$
\begin{aligned}
X_{\lceil\log p\rceil+\tau} & =\left\{3+4 i: 0 \leq i \leq 2^{\lceil\log p\rceil-2}+\tau p / 4-1\right\} \\
& \cup\left\{N-3-4 i: 0 \leq i \leq 2^{\lceil\log p\rceil-2}+\tau p / 4-2\right\} .
\end{aligned}
$$

Now consider the matchings $M=\{(v, v+1): v$ is even $\}$ and $M^{\prime}=\{(v, v+3)$ : $v$ is even $\}$; it is easy to see that gossiping can be completed in two more rounds by exchanging calls during rounds $\lceil\log p\rceil+\left\lceil\frac{N-2^{\lceil\log p\rceil}}{p}\right\rceil$ and $\lceil\log p\rceil+\left\lceil\frac{N-2^{\lceil\log p\rceil}}{p}\right\rceil+1$ along the edges of $M$ and $M^{\prime}$ if $N=1(\bmod 4)$ or the edges of $M^{\prime}$ and $M$ if $N=3(\bmod 4)$, respectively.

3.4. Hypercube. In the next theorem we shall determine $g(p, G)$ for any $p$ when the graph $G$ is the $d$-dimensional hypercube $H_{d}$ with $2^{d}$ nodes.

THEOREM 3.6. For each integer $p<2^{d}, \quad g\left(p, H_{d}\right)=\lceil\log p\rceil+\left\lceil\frac{1}{p}\left(2^{d}-2^{\lceil\log p\rceil}\right)\right\rceil$.

Proof. The lower bound follows from Lemma 3.1. We now prove the matching upper bound. Let $p$ be fixed. Denote by $t_{d}$ the minimum integer such that $I\left(p, t_{d}\right) \geq$ $2^{d}$, where $I\left(p, t_{d}\right)$ is given in (11). We shall show that there exists a gossiping protocol that requires $t_{d}$ rounds. Notice that $t_{d}=\lceil\log p\rceil+\left\lceil\frac{1}{p}\left(2^{d}-2^{\lceil\log p\rceil}\right)\right\rceil$.

The proof is by induction on $d$. The assertion is trivially true for $d=1$; suppose that there exists a gossiping protocol in $H_{d}$ that takes $t_{d}$ rounds to be completed and that satisfies the additional property that after any round $t \leq t_{d}-1$ each vertex knows exactly $I(p, t)$ packets. We shall exhibit a gossiping protocol in $H_{d+1}$ that takes $t_{d+1}$ rounds to be completed and that also satisfies the aforesaid additional property.

Case 1: $I\left(p, t_{d}\right)=2^{d}$. This implies that in the last round of the gossiping protocol in $H_{d}$-the $t_{d}$ th - each vertex must receive exactly $\min \left\{p, 2^{d-1}\right\}$ packets. Consider the following protocol in the $(d+1)$-dimensional hypercube $H_{d+1}$ : split $H_{d+1}$ into two hypercubes of dimension $d$ according to the value of its $(d+1)$ th dimension; during the first $t_{d}$ rounds gossip separately in each $d$ dimensional subcube according to the protocol whose existence is guaranteed by the induction hypothesis. After $t_{d}$ rounds each vertex has received all the information of the subcube it belongs to; i.e., according to the hypothesis of this case, each vertex has received exactly $I\left(p, t_{d}\right)=2^{d}$ packets. Now, in successive rounds, exchange packets along dimension $d+1$ in $H_{d+1}$ by sending either all $2^{d}$ packets in one round, if $p>2^{d}$, or $p$ packets per round except in the last one, where one sends $2^{d}-p\left\lfloor 2^{d} / p\right\rfloor$ (if nonzero) packets. It is clear that this protocol requires $t_{d+1}$ rounds to be completed. Moreover, for each $t$, with $0 \leq t \leq\left\lfloor 2^{d} / p\right\rfloor$, after round $t_{d}+t \leq t_{d+1}-1$ each node in $H_{d+1}$ knows exactly $I\left(p, t_{d}\right)+p t=I\left(p, t_{d}+t\right)$ packets. Hence the protocol for $H_{d+1}$ satisfies the inductive hypothesis.

Case 2: $I\left(p, t_{d}\right)>2^{d}$. This implies that $p<2^{d-1}$; otherwise it is easy to check that one would have $t_{d}=d$ and $I\left(p, t_{d}\right)=1+\sum_{i} 2^{i-1}=2^{d}$. Consider the protocol in $H_{d}$ whose existence is implied by the induction hypothesis. By inductive hypothesis at round $t_{d}-1$ each vertex has received $I\left(p, t_{d}-1\right)$ packets, and in the last round, receives $\alpha$ packets, with $\alpha<p$; otherwise, we 
would be again in Case 1 .

Let $\mathcal{M}=\cup_{i=1}^{2^{d-1}}\left(x_{i}, y_{i}\right)$ be the perfect matching used in the last round, i.e., the round $t_{d}$, of the protocol on $H_{d}$, and let $A_{i}$ (resp., $B_{i}$ ) be the set of new packets that $x_{i}$ (resp., $y_{i}$ ) receives in this last round. Note that $A_{i} \cap B_{i}=\emptyset$ and $\left|A_{i}\right|=\left|B_{i}\right|=\alpha$. For what follows, let $C_{i}$ and $D_{i}$ be two sets of packets such that $\left|C_{i}\right|=\left|D_{i}\right|=p-\alpha$ and $C_{i} \cap A_{i}=\emptyset, D_{i} \cap A_{i}=\emptyset, C_{i} \cap B_{i}=\emptyset$, $D_{i} \cap B_{i}=\emptyset$, and $C_{i} \cap D_{i}=\emptyset$. Such sets exist since $\left|A_{i}\right|+\left|B_{i}\right|+\left|C_{i}\right|+\left|D_{i}\right|=$ $2 p<2{ }^{d}$. Consider now the following gossiping protocol in $H_{d+1}$. Split $H_{d+1}$ according to the value of the $(d+1)$ th dimension in two subcubes $H_{d}$ and $H_{d}^{\prime}$ of dimension $d$; during the first $t_{d}-1$ rounds, gossip in $H_{d}$ and $H_{d}^{\prime}$ separately. At the end of this phase each vertex knows $2^{d}-\alpha$ packets. Now, for each node $x$ in $H_{d}$, denote by $x^{\prime}$ its neighbor in $H_{d}^{\prime}$. In the next round, exchange $p$ packets along dimension $d+1$ in such a way that $x_{i}$ (resp., $y_{i}, x_{i}^{\prime}, y_{i}^{\prime}$ ) sends to $x_{i}^{\prime}$ (resp., $\left.y_{i}^{\prime}, x_{i}, y_{i}\right) p$ packets including $C_{i}$ (resp., $\left.D_{i}, C_{i}^{\prime}, D_{i}^{\prime}\right)$ and not $D_{i}$ (resp., $\left.C_{i}, D_{i}^{\prime}, C_{i}^{\prime}\right)$.

In the next round, exchange $p$ packets along the matching $\mathcal{M}$ in such a way that $x_{i}$ (resp., $y_{i}$ ) sends to $y_{i}$ (resp., $x_{i}$ ) packets in $B_{i} \cup C_{i}^{\prime}$ (resp., $A_{i} \cup D_{i}^{\prime}$ ), and $x_{i}^{\prime}$ (resp., $y_{i}^{\prime}$ ) sends to $y_{i}^{\prime}$ (resp., $x_{i}^{\prime}$ ) all packets in $B_{i}^{\prime} \cup C_{i}$ (resp., $A_{i}^{\prime} \cup D_{i}$ ). After the above $t_{d}+1$ rounds we are sure that each vertex $x_{i}$ (resp., $x_{i}^{\prime}$ ) knows all the packets of the subcube it belongs to, and so we can finish the protocol by sending packets along dimension $d+1$ in such a way that $p$ new packets are received during each round (except possibly the last final round). Therefore, for each $t$, with $1 \leq t \leq 1+\left\lfloor 2^{d} / p\right\rfloor$, each node in $H_{d+1}$ after round $t_{d}+t-1 \leq t_{d+1}-1$ knows exactly $I\left(p, t_{d}-1\right)+p t=I\left(p, t_{d}+t-1\right)$ packets. Hence this protocol in $H_{d+1}$ satisfies all the induction hypothesis.

Remark 3.3. It is worth pointing out that the obvious inequality

$$
g_{H_{1}}(p, G) \leq 2 g_{F_{1}}(p, G)
$$

and the above theorem allow us to improve the upper bound on $g_{H_{1}}\left(p, H_{d}\right)$ given by Theorem 4 of [4] for all values of $p$ which are not powers of two. Indeed, the authors of [4] have $g_{H_{1}}\left(p, H_{d}\right) \leq 2 d+2^{d+1} / p-2 / p$, while from Theorem 3.6 and (21), we get Theorem 3.7.

Theorem 3.7. For each integer $p<2^{d}$,

$$
g_{H_{1}}\left(p, H_{d}\right) \leq 2\lceil\log p\rceil+2\left\lceil\frac{1}{p}\left(2^{d}-2^{\lceil\log p\rceil}\right)\right\rceil .
$$

4. $p$-optimal graphs. In this section we consider the problem of estimating the minimum possible number of edges in any graph in which gossiping can be performed in the minimum possible number of rounds. We consider only networks with an even number of nodes. More formally, for any even integer $n$ and integer $p$ such that $2^{\lceil\log p\rceil} \leq n$, let us denote by $g(p, n)$ the minimum gossiping time of any graph with $n$ nodes, that is (cf. Theorem 3.3),

$$
g(p, n):=\min _{G:|V(G)|=n} g(p, G)=\lceil\log p\rceil+\left\lceil\frac{n-2^{\lceil\log p\rceil}}{p}\right\rceil,
$$

and by $\mathcal{M}(p, n)$, the quantity 


$$
\mathcal{M}(p, n):=\min \{m: \text { there exists } G=(V, E) \text { with }|V|=n,|E|=m, g(p, G)=g(p, n)\} .
$$

Our objective is to find significant bounds on the function $\mathcal{M}(p, n)$. From a practical point of view, an interconnection network $G$ having gossiping time $g(p, G)=g(p, n)$ and $\mathcal{M}(p, n)$ edges represents the most economical network, if our main concern is the number of communication lines, that still preserves the communication capabilities of the complete graph, as far as gossiping is concerned. The analogous problem of estimating the minimum possible number of edges in a network in which broadcasting can be performed in minimum time has been extensively studied (see [5, 13] and references therein quoted). Estimating $\mathcal{M}(p, n)$ seems a much harder task. Even when $p$ is unbounded, only few results are known [21].

DEFINITION 4.1. Given a graph $G(V, E)$ on $n$ nodes and an integer $p$ such that $2^{\lceil\log p\rceil} \leq n$ we say that $G$ is $p$-optimal if $g(p, G)=g(p, n)$ and $|E|=\mathcal{M}(p, n)$, that is, if $G$ is a sparsest graph among all the graphs with $n$ nodes and minimum gossiping time $g(p, n)$.

We first consider the special cases $p=1$ and $p=2$ that admit a very simple solution, and afterwards we consider the general case, that is, $p \geq 3$.

4.1. Sending $p \leq 2$ items per round. We have shown in sections 2.2 and 3.2 that for the ring $C_{n}$ on $n$ nodes

$$
g\left(1, C_{n}\right)=g(1, n)=2\left\lceil\frac{n}{2}\right\rceil-1 \text { and } g\left(2, C_{n}\right)=g(2, n)= \begin{cases}n / 2 & \text { if } n \text { is even } \\ (n+3) / 2 & \text { if } n \text { is odd }\end{cases}
$$

Consider any connected graph (tree) $G$ with $n$ nodes and $m \leq n-1$ edges. The lower bound given in Corollary 2.2 tells us that $g(1, G) \geq 2 n-3$. Moreover, it is easy to verify that the inequality $g(1, G) \leq 2 g(2, G)-1$ holds. The above two inequalities imply that $g(1, G)>g(1, n)=n-1$ for each $n \geq 3$ and $g(2, G) \geq(g(1, G)+1) / 2 \geq$ $(2 n-2) / 2=n-1 \geq g(2, n)$ for each $n \geq 2$ with $n \neq 3$. It is easy to see that $P_{3}$ is also optimal for $p=2$. We have then proved the following theorem.

TheOREM 4.1. $\mathcal{M}(1,2)=\mathcal{M}(2,2)=1, \mathcal{M}(1,3)=3, \mathcal{M}(2,3)=2$, and for each $n \geq 4$

$$
\mathcal{M}(1, n)=\mathcal{M}(2, n)=n
$$

4.2. Sending $p \geq 3$ items per round. In this section we study $p$-optimal graphs for $p \geq 3$. We recall that such graphs are to be sought among those graphs having gossiping time equal to $g(p, n)$. Let us first recall that for each $p \geq 3$ the ring $C_{n}$ is not $p$-optimal; indeed, from the results of section 3.2 we have $\min _{q} g\left(q, C_{n}\right)=$ $g\left(2, C_{n}\right)>g(p, n)$.

We have proved in section 3.4 that the hypercube $H_{d}$ has minimum gossiping time for each value of $p$; moreover, it was shown in [21] that $H_{d}$ is $p$-optimal for each $p \geq 2^{d-1}$ (equivalently, for $p$ unbounded); that is, $H_{d}$ has the minimum number of edges among all the networks with gossiping time $g\left(\infty, 2^{d}\right)=d$. A natural question is whether the hypercube is $p$-optimal for other values of $p<2^{d-1}$. The results of section 4.3 will imply a negative answer to the above question.

Let $d(p, n)$ be the minimum possible degree a node can have in any $p$-optimal graph on $n$ nodes. 
THEOREM 4.2. $d(p, n) \geq\lfloor\log p\rfloor+1-\left\lfloor\log \left(\left\lceil\frac{n-2^{\lceil\log p\rceil}}{p}\right\rceil p-n+2^{\lceil\log p\rceil}+1\right)\right\rfloor$.

Proof. Denote by $r(p, t)$ the maximum number of items a node can receive with a call made at round $t$, and by $I(p, t)=1+\sum_{i=1}^{t} r(p, i)$, the maximum possible number of items a node can have received by round $t$. We recall that $I(p, 0)=r(p, 1)=1$ and

$$
r(p, t)=\max \left\{2^{t-1}, p\right\}
$$

and

$$
I(p, t)= \begin{cases}2^{t} & \text { if } t \leq\lfloor\log p\rfloor+1 \\ 2^{\lfloor\log p\rfloor+1}+(t-\lfloor\log p\rfloor-1) p & \text { if } t>\lfloor\log p\rfloor+1\end{cases}
$$

Fix any gossiping protocol $\mathcal{P}$ that completes in $g(p, n)$ rounds. We denote by $r(p, t, v)$ the number of items node $v$ receives at round $t$ of $\mathcal{P}$ and let

$$
I(p, t, v)=1+\sum_{i=1}^{t} r(p, i, v)
$$

obviously $r(p, t, v) \leq r(p, t)$ and $I(p, t, v) \leq I(p, t)$ for each $t=1, \ldots, g(p, n)$.

In order to prove the desired lower bound on $d(p, n)$ we show that any node has to make calls with at least $\lfloor\log p\rfloor+1-\left\lfloor\log \left(\left\lceil\frac{n-2^{\lceil\log p\rceil}}{p}\right\rceil p-n+2^{\lceil\log p\rceil}+1\right)\right\rfloor$ different neighbors during the first $\lfloor\log p\rfloor+1$ rounds of the protocol $\mathcal{P}$.

Fix a node $v$ and suppose that $v$ communicates with $\lfloor\log p\rfloor+1-\ell$ different neighbors during the first $\lfloor\log p\rfloor+1$ rounds of $\mathcal{P}$. This means that there exist $\ell$ rounds, say $\tau_{1}, \ldots, \tau_{\ell}$, such that for each $i=1, \ldots, \ell$ at round $\tau_{i}, v$ is either idle or makes a call with a node that will communicate again with $v$ at some round $\delta_{i}$ with $\tau_{i}<\delta_{i} \leq\lfloor\log p\rfloor+1$; we can bound $r\left(p, \tau_{i}, v\right)$ as follows.

i) If $v$ does not participate in any call at round $\tau_{i}$, then $r\left(p, \tau_{i}, v\right)=0$.

ii) If $v$ makes calls with a particular node, say $w$, at both rounds $\tau_{i}$ and $\delta_{i}$, then at time $\delta_{i}$ node $v$ will not receive again what it received at time $\tau_{i}$ from $w$, nor will it receive what it sent to $w$ at time $\tau_{i}$. Therefore, $r\left(p, \delta_{i}, v\right) \leq$ $I\left(p, \delta_{i}-1, w\right)-r\left(p, \tau_{i}, v\right)-r\left(p, \tau_{i}, w\right) ;$ that is,

$$
r\left(p, \tau_{i}, v\right)+r\left(p, \delta_{i}, v\right) \leq I\left(p, \delta_{i}-1, w\right) \leq 2^{\delta_{i}-1}=r\left(p, \delta_{i}\right)
$$

By i) and ii) we get that for each round $t \geq\lfloor\log p\rfloor+1$,

$$
\begin{aligned}
I(p, t, v) & =1+\sum_{i-1}^{t} r(p, i, v) \leq I(p, t)-\sum_{i=1}^{\ell} 2^{\tau_{i}-1} \\
& =2^{\lfloor\log p\rfloor+1}+(t-\lfloor\log p\rfloor-1) p-\sum_{i=1}^{\ell} 2^{\tau_{i}-1} .
\end{aligned}
$$

Recalling that $n$ is even and the graph has minimum gossiping time $g(p, n)=\lceil\log p\rceil+$ $\left\lceil\frac{n-2^{\lceil\log p\rceil}}{p}\right\rceil=\lfloor\log p\rfloor+1+\left\lceil\frac{n-2^{\lfloor\log p\rfloor+1}}{p}\right\rceil$, the following inequality must be satisfied:

$$
n \leq I(p, g(p, n))-\sum_{i=1}^{\ell} 2^{\tau_{i}-1}=2^{\lfloor\log p\rfloor+1}-\sum_{i=1}^{\ell} 2^{\tau_{i}-1}+\left\lceil\frac{n-2^{\lfloor\log p\rfloor+1}}{p}\right\rceil p .
$$


Noticing that $\sum_{i=1}^{\ell} 2^{\tau_{i}-1} \leq \sum_{i=1}^{\ell} 2^{i-1}=2^{\ell}-1$, we get

$$
\ell \leq\left\lfloor\log \left(\left\lceil\frac{n-2^{\lfloor\log p\rfloor+1}}{p}\right\rceil p-n+2^{\lfloor\log p\rfloor+1}+1\right)\right\rfloor
$$

and the desired bound on $d(n, p)$ follows.

4.3. A family of graphs with $O\left(\frac{n}{2} \log p\right)$ edges. In section 3.3 we have proved that $g\left(p, K_{n}\right)=g(p, n)$ for any even $n$ and any $p$. Moreover, it is easy to see that in order to implement the gossiping protocol of Figure 9 , only $O\left(n\left(\frac{n}{p}+\log p\right)\right)$ edges of $K_{n}$ are needed. This implies that $\mathcal{M}(p, n)=O\left(n\left(\frac{n}{p}+\log p\right)\right)$. Actually, we can prove a much better bound. We will construct for any $p$ and even $n$ a graph $G_{p, n}$ with $n$ nodes, $n(\lceil\log p\rceil+1) / 2$ edges, and optimal gossiping time $g\left(p, G_{p, n}\right)=g(p, n)$.

Let $p$ be an even integer and define the sequence of integers $\mathbf{s}_{p}$ as follows: $\mathbf{s}_{2}=$ $(-1,1)$ and for each $p=2^{m}+q$ with $q \leq 2^{m}$, if $\mathbf{s}_{2^{m}}=\left(s_{1}, \ldots, s_{m+1}\right)$, then

$$
\mathbf{s}_{p}=\left(s_{1}, \ldots, s_{m+1}, s_{m+2}\right) \quad \text { with } s_{m+2}= \begin{cases}p+s_{m+1} & \text { if } m \text { is even } \\ -\left(p-s_{m+1}\right) & \text { if } m \text { is odd }\end{cases}
$$

If $p$ is odd define $\mathbf{s}_{p}=\mathbf{s}_{p+1}$.

Example 4.1. $\mathbf{s}_{2}=(-1,1), \mathbf{s}_{3}=\mathbf{s}_{4}=(-1,1,-3), \mathbf{s}_{5}=\mathbf{s}_{6}=(-1,1,-3,3)$, $\mathbf{s}_{7}=\mathbf{s}_{8}=(-1,1,-3,5), \mathbf{s}_{9}=\mathbf{s}_{10}=(-1,1,-3,5,-5), \mathbf{s}_{11}=\mathbf{s}_{12}=(-1,1,-3,5,-7)$. Let the node set be $V_{n}=\{0,1, \ldots, n-1\}$. All operations on nodes will be performed modulo $n$. Define the matching

$$
M_{p, n}(t)=\left\{\left(v, v+s_{t}\right) \mid v \in V_{n} \text { is odd }\right\} \quad \text { for } t=1 \ldots,\lceil\log p\rceil+1,
$$

and the graph $G_{p, n}=\left(V_{n}, E_{p, n}\right)$ with $E_{p, n}=\cup_{t=1}^{[\log p\rceil+1} M_{p, n}(t)$; Figure 11 shows $G_{6,14}$.

One can check that at the end of the algorithm Gossiping $\left(G_{p, n}\right)$, given in Figure 12 , any node knows the packets of all the other nodes in $G_{p, n}$. Therefore, using Theorem 4.2, we get Theorem 4.3.

THEOREM 4.3. For each integer $p$ and even integer $n \geq 2^{\lceil\log p\rceil}$

$$
\begin{aligned}
\frac{n}{2}\left(\lfloor\log p\rfloor+1-\left\lfloor\log \left(\left\lceil\frac{n-2^{\lceil\log p\rceil}}{p}\right\rceil p-n+2^{\lceil\log p\rceil}+1\right)\right\rfloor\right) \\
\quad \leq \mathcal{M}(p, n) \leq \frac{n}{2}(\lceil\log p\rceil+1) .
\end{aligned}
$$

Corollary 4.1. For each $p \geq 2$ and even integer $n$ such that $n-2^{\lceil\log p\rceil}$ is a multiple of $p$,

$$
\frac{n}{2}(\lfloor\log p\rfloor+1) \leq \mathcal{M}(p, n) \leq \frac{n}{2}(\lceil\log p\rceil+1)
$$

CoROllary 4.2. For each integer $q \geq 1$ and integer $r \geq 2$,

$$
\mathcal{M}\left(2^{q}, r 2^{q}\right)=r 2^{q-1}(q+1) .
$$

It is possible to improve the lower bound given in Theorem 4.2 proving that $d\left(2^{q}-1, n\right) \geq q+1-\left\lfloor\log \left(\left\lceil(n-1) /\left(2^{q}-1\right)\right\rceil\left(2^{q}-1\right)-n+2\right)\right\rfloor$, which together with Theorem 4.3 implies the following corollary.

COROLlary 4.3. For each integer $q \geq 1$ and odd integer $r$,

$$
\mathcal{M}\left(2^{q}-1, r\left(2^{q}-1\right)+1\right)=\left(r\left(2^{q}-1\right)+1\right)(q+1) / 2 .
$$



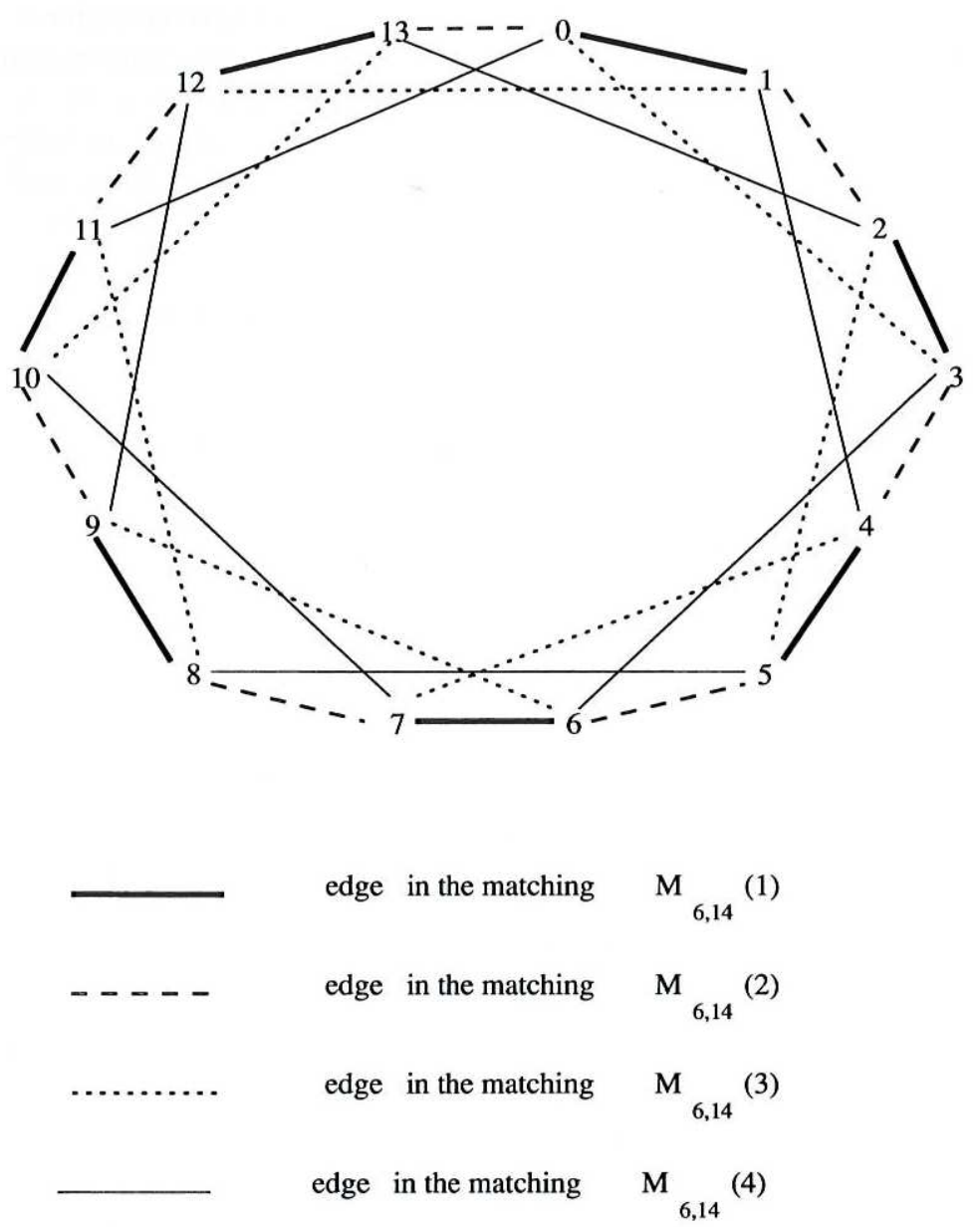

FIG. 11.

\section{$\operatorname{Gossiping}\left(G_{p, n}\right)$}

Let $\mathbf{s}_{p}=\left(s_{1}, \ldots, s_{\lceil\log p\rceil+1}\right)$;

At round $t, t=1, \ldots,\lceil\log p\rceil+1, \quad$ each node sends $\max \left\{2^{t-1}, p\right\}$ new items to its neighbor in $M_{p, n}(t)$;

At round $t=\lceil\log p\rceil+1+\tau, \tau=1, \ldots, g(p, n)-\lceil\log p\rceil-1, \quad$ consider the matching

$$
M_{p, n}(t)= \begin{cases}M_{p, n}(\lceil\log p\rceil) & \text { if } \tau \text { is odd } \\ M_{p, n}(\lceil\log p\rceil+1) & \text { if } \tau \text { is even }\end{cases}
$$

then each node sends $p$ new items (or fewer than $p$ in the last round) to its neighbor in $M_{p, n}(t)$.

FIG. 12. Gossiping algorithm in $G_{p, n}$. 
5. Concluding remarks and open problems. We have considered the problem of gossiping in communication networks under the restriction that communicating nodes can exchange up to a fixed number $p$ of packets at each round. In the extremal case $p=1$ we have given optimal algorithms to perform gossiping in several classes of graphs, including Hamiltonian graphs, paths, complete $k$-ary trees, and complete bipartite graphs. For arbitrary graphs we gave asymptotically matching upper and lower bounds.

In the case of arbitrary $p$ we have determined the optimal number of communication rounds to perform gossiping under this hypothesis for complete graphs, hypercubes, rings, paths, and complete bipartite graphs $K_{r, r}$.

Several open problems remain in the area. We list the most important of them here.

- It would be interesting to determine the computational complexity of computing $g_{F_{1}}(1, G)\left(g_{F_{1}}(p, G)\right)$ for general graphs; it is very likely that it is NP-hard. (We know that computing $g_{F_{1}}(\infty, G)$ is NP-hard; see [20].)

- We have left open the problem of determining the gossiping time $g_{F_{1}}\left(1, G_{t, s}\right)$, and more generally $g_{F_{1}}\left(p, G_{t, s}\right)$, of rectangular grids $G_{t, s}$ with both $t$ and $s$ odd. We know from Corollary 2.1 that $g_{F_{1}}\left(1, G_{t, s}\right) \geq s t+1$. Does equality hold? We can prove that $g_{F_{1}}\left(1, G_{3,3}\right)=10$. A general upper bound on $g_{F_{1}}\left(1, G_{t, s}\right)$ can be obtained by observing that $G_{t, s}=P_{t} \times P_{s}$, where $P_{t}$ and $P_{s}$ are the paths on $t$ and $s$ nodes, respectively, and $\times$ denotes the cartesian graph product. Now, given two graphs $G=(V, E)$ and $H=$ $(W, F)$, it is easy to see that $g_{F_{1}}(1, G \times H) \leq \min \left\{g_{F_{1}}(1, G)+|V| g_{F_{1}}(1, H), g_{F_{1}}(1, H)+\right.$ $\left.|W| g_{F_{1}}(1, G)\right\}$, which, together with Corollary 2.5 , immediately gives $g_{F_{1}}\left(1, G_{t, s}\right) \leq$ $2 t s-3-\max \{t, s\}$.

- We know from (4) that for any graph $G$ with $n$ vertices one has $g_{F_{1}}(1, G) \geq n$ if $n$ is odd, $g_{F_{1}}(1, G) \geq n-1$ if $n$ is even, and from Theorem 2.1, we get that the equality holds for Hamiltonian graphs. It would be interesting to characterize the class of graphs for which this lower bound is tight. We know from the results of section 2.5 that this class is larger than the class of the Hamiltonian graphs.

- In view of the possible NP-hardness of computing $g(p, G)$ for arbitrary graphs, it would be interesting to design efficient algorithms to compute gossiping protocols that complete in time "close" to $g(p, G)$. Such algorithms have recently been provided for $g(\infty, G)$ (see $[14,23])$. However, the techniques used there do not seem to apply to the case of bounded $p$.

- We have given fairly tight bounds on the function $\mathcal{M}(p, n)$. It would be interesting to study the analogous quantity $\mathcal{M}_{c}(p, n)$ equal to the minimum number of edges in any graph in which gossiping can be performed in quasi-optimal time $g(p, n)+c$, where $c$ is a small constant. In particular, we ask whether $\mathcal{M}_{1}(p, n)=O(n)$.

Acknowledgments. The authors wish to thank R. Ravi for the proof of Theorem 2.3. They also want to thank Levon Khachatrian and Jonny Bond for interesting discussions. The first author would like to thank the Dipartimento di Informatica ed Applicazioni, Università di Salerno, Italy, where part of this research was conducted, for inviting him.

\section{REFERENCES}

[1] N. Alon, F.R.K. Chung, AND R.L. Graham, Routing permutations on graphs via matchings, in Proc. 25th ACM Symposium on the Theory of Computing (STOC '93), San Diego, CA, 1993, pp. 583-591. 
[2] B. AlsPaCH, The classification of Hamiltonian generalized Petersen graphs, J. Combin. Theory Ser. B, 34 (1983), pp. 293-312.

[3] A. BAGCHI, E.F. SCHMEICHEL, AND S.L. HAKIMI, Sequential information dissemination by packets, Networks, 22 (1992), pp. 317-333.

[4] A. BAGChI, E.F. SchmeIChel, AND S.L. HAKImI, Parallel information dissemination by packets, SIAM J. Comput., 23 (1994), pp. 355-372.

[5] J.-C. Bermond, P. Fraignaud, AND J. Peters, Antepenultimate broadcasting, Networks, 25 (1995), pp. 125-137.

[6] D. P. Bertsekas and J. N. Tsitsiklis, Parallel and Distributed Computation: Numerical Methods, Prentice-Hall, Englewood Cliffs, NJ, 1989.

[7] B. S. Chlebus, K. Diks, AND A. PelC, Optimal gossiping with short unreliable messages, Disc. Appl. Math., 53 (1994), pp. 15-24.

[8] K. DIKS AND A. PELC, Efficient gossiping by packets in networks with random faults, SIAM J. Disc. Math., 9 (1996), pp. 7-18.

[9] G. Fox, M. Johnsson, G. Lyzenga, S. Otto, J. Salmon, and D. Walker, Solving Problems on Concurrent Processors, Volume I, Prentice-Hall, Englewood Cliffs, NJ, 1988.

[10] P. Fraignaud AND E. LAzARD, Methods and problems of communication in usual networks, Disc. Appl. Math., 53 (1994), pp. 79-134.

[11] S. FUJITA, Gossiping in mesh-bus computers by packets with bounded length, IPS Japan SIGAL, 36 (1993), pp. 41-48.

[12] M. FÜRER AND B. RAGHAVACHARI, Approximating the minimum degree spanning tree to within one from the optimal degree, in Proc. Third Annual ACM-SIAM Symposium on Discrete Algorithms (SODA '92), Orlando, FL, 1992, pp. 317-324.

[13] M. GRIGNI AND D. PELEG, Tight bounds on minimum broadcast networks, SIAM J. Disc. Math., 5 (1992), pp. 207-222.

[14] G. KoRTSARZ AND D. PELEG, Approximation algorithms for minimum time broadcast, SIAM J. Disc. Math., 8 (1995), pp. 401-427.

[15] S. M. Hedetniemi, S. T. Hedetniemi, And A. Liestman, A survey of gossiping and broadcasting in communication networks, Networks, 18 (1988), pp. 129-134.

[16] A. Hily AND D. SotTeAU, Communications in Bus Networks, in Parallel and Distributed Computing, Lectures Notes in Computer Science 805, M. Cosnard, A. Ferreira, and J. Peters, eds., Springer-Verlag, New York, 1994, pp. 197-206.

[17] J. Hromkovič, R. Klasing, B. Monien, AND R. Peine, Dissemination of Information in Interconnection Networks (Broadcasting and Gossiping), in Combinatorial Network Theory, F. Hsu and D.-Z. Du, eds., Kluwer Academic Publishers, Norwell, MA, 1995, pp. 125-212.

[18] S. L. JohNSSON AND C. T. Ho, Matrix multiplication on Boolean cubes using generic communication primitives, in Parallel Processing and Medium-Scale Multiprocessors, A. Wouk, ed., SIAM, Philadelphia, 1989, pp. 108-156.

[19] D. W. KRumme, Fast gossiping for the hypercube, SIAM J. Comput., 21 (1992), pp. 365-380.

[20] D. W. Krumme, K. N. Venkataraman, and G. Cybenko, Gossiping in minimal time, SiAM J. Comput., 21 (1992), pp. 111-139.

[21] R. LABHAN, Some minimum gossip graphs, Networks, 23 (1993), pp. 333-341.

[22] R. RAVI, private communication.

[23] R. RAVI, Rapid rumor ramification: Approximating the minimum broadcasting time, in Proc. 35th Annual Symposium on Foundations of Computer Science (FOCS '94), 1994, pp. 202213.

[24] J. DE Rumeur, Communication dans les Reseaux de Processeur, Masson, Paris, 1994. 